\title{
Signatures of two distinct driving mechanisms in the evolution of coronal mass ejections in the lower corona
}

\author{
C. Loesch, ${ }^{1}$ M. Opher, ${ }^{2}$ M. V. Alves, ${ }^{1}$ R. M. Evans, ${ }^{2}$ and W. B. Manchester ${ }^{3}$ \\ Received 19 April 2010; revised 12 January 2011; accepted 21 January 2011; published 13 April 2011.
}

[1] We present a comparison between two simulations of coronal mass ejections

(CMEs), in the lower corona, driven by different flux rope mechanisms presented in the literature. Both mechanisms represent different magnetic field configurations regarding the amount of twist of the magnetic field lines and different initial energies. They are used as a "proof of concept" to explore how different initialization mechanisms can be distinguished from each other in the lower corona. The simulations are performed using the Space Weather Modeling Framework (SWMF) during solar minimum conditions with a steady state solar wind obtained through an empirical approach to mimic the physical processes driving the solar wind. Although the two CMEs possess different initial energies (differing by an order of magnitude) and magnetic configurations, the main observables such as acceleration, shock speed, Mach number, and $\theta_{B n}$ (the angle between the shock normal and the upstream magnetic field) present very similar behavior between 2 and $6 R_{\odot}$. We believe that through the analysis of other quantities, such as sheath width and postshock compression (pileup and shock indentation compressions), the effect of different magnetic configurations and initializations can be distinguished. We discuss that coronal models that employ a reduced value of polytropic index $(\gamma)$ may significantly change the energetics of the CME and that the background solar wind plays an important role in the CMEs' shock and sheath evolution.

Citation: Loesch, C., M. Opher, M. V. Alves, R. M. Evans, and W. B. Manchester (2011), Signatures of two distinct driving mechanisms in the evolution of coronal mass ejections in the lower corona, J. Geophys. Res., 116, A04106, doi:10.1029/2010JA015582.

\section{Introduction}

[2] Coronal mass ejections (CMEs) are large-scale expulsions of plasma and magnetic field from the solar corona to the interplanetary space. During a large CME event, $\sim 10^{16} \mathrm{~g}$ of coronal material with energies of $\sim 10^{32}$ ergs are ejected from the Sun [Hundhausen, 1997; Vourlidas et al., 2002]. While accelerating away from the Sun, CMEs present speeds between few tens up to $\sim 2500 \mathrm{~km} / \mathrm{s}$. CMEs with speeds exceeding the magnetosonic speed can drive fast shocks ahead of them. CME-driven fast shocks are able to accelerate charged particles up to very high energies ( $\mathrm{GeV} /$ nucleon) [Wang and Wang, 2006].

[3] At the present time, CMEs magnetic structure and triggering mechanisms are still not thoroughly understood. Many models, analytical and numerical, have been developed describing possible CMEs onset topologies for the coronal magnetic field [e.g., Forbes and Priest, 1995; Linker and

\footnotetext{
${ }^{1}$ Laboratório Associado de Plasma, Instituto Nacional de Pesquisas Espaciais, São José dos Campos, Brazil.

${ }^{2}$ Department of Physics and Astronomy, George Mason University, Fairfax, Virginia, USA.

${ }^{3}$ Center for Space Environment Modeling, University of Michigan, Ann Arbor, Michigan, USA.

Copyright 2011 by the American Geophysical Union. 0148-0227/11/2010JA015582
}

Mikić, 1995; Antiochos et al., 1999; Odstrcil et al., 2002; Roussev et al., 2004]. One topology commonly assumed for the coronal magnetic field is that of a flux rope, where CMEs can occur from helical kink or toroidal instabilities [e.g., Chen, 1989, 1996; Forbes and Priest, 1995; Gibson and Low, 1998; Titov and Démoulin, 1999; Wu et al., 1999, 2001; Roussev et al., 2003a; Manchester et al., 2004a, 2004b, 2004c; Torok and Kliem, 2005]. Another initiation model assumes that a magnetic arcade is driven to erupt by shearing motions and magnetic reconnection [e.g., Linker and Mikić, 1995; Odstrcil et al., 2002; Roussev et al., 2004; Jacobs et al., 2009]. The breakout model of Antiochos et al. [1999] assumes a quadrupolar configuration that allows reconnection to occur at the top of the arcade allowing significantly faster eruptions. Manchester [2007] examined dynamic shear flows driven by the Lorentz force, which have been proposed as an explanation for CME initiation in both, arcades [Manchester, 2003] and emerging flux ropes [Manchester et al., 2004a].

[4] In this paper we aim to investigate the role of the magnetic field configuration in CMEs evolution in the lower corona, and how the signatures of shocks vary as a function of that. We use two distinct analytical flux rope mechanisms: those of Gibson and Low [1998, hereafter GL98] and Titov and Démoulin [1999, hereafter TD99]. The simulations are performed with the Space Weather Modeling Framework 
(SWMF)[Tóth et al., 2005]. We choose the GL98 and TD99 models since they can represent, according to chosen appropriate initial conditions, different magnetic field configurations regarding the amount of twist of the magnetic field lines. They have also been successfully used within SWMF, as in work by Manchester et al. [2004a], using the GL98 model, they found that the flux rope expands rapidly, driving a strong shock ahead of it along with large amounts of plasma mimicking a CME. Studies by, e.g., Lugaz et al. [2007], Liu et al. [2008a], and Lugaz et al. [2009] initiated their CMEs using a modified version of TD99, also used in this paper and addressed in section 2 .

[5] In the GL98 model, an axisymmetric, spherical toroidal flux rope is distorted into a teardrop shape, a configuration that gives the flux rope system sufficient free energy for an eruption. In the TD99 model original version, a flux rope (above the surface) is prescribed by a given toroidal current and is in equilibrium with a background field generated by an infinite line current and two point charges underneath the photosphere. In our simulations we present a modified version of the TD99 model, where the flux rope is not in equilibrium with the background field. And, the background field generated by the point charges and the infinite line current are not included [e.g., Lugaz et al., 2007; Liu et al., 2008a; Lugaz et al., 2009]. Our flux rope solution is superimposed on the ambient magnetic field obtained for the steady state solar wind. In our simulations the TD99 model is representative of a much more highly twisted flux rope than the GL98 one due to our choice of initial parameters. We used both mechanisms as a "proof of concept" to explore how different initialization mechanisms can be distinguishable in the lower corona.

[6] For our purposes, we utilize the solar corona (SC) component of SWMF. The steady state solar wind utilizes a variable polytropic index [Cohen et al., 2007, 2008], that heats the solar wind with distance (for estimation of the heating see Evans et al. [2009]). We fixed the profile of the polytropic index after the initialization of the CME therefore neglecting any exchange of energy between the CME and the solar wind.

[7] We compare the signatures of the two driving mechanisms during the CME propagation in the lower corona (from $\sim 2 R_{\odot}$ to $6 R_{\odot}$, where $R_{\odot}$ is the solar radius). We set the highest numerical resolution through the center of the CME. For this reason we analyze the signatures only at the nose of the CME. This work can serve as a prototype to explore signatures of initiation mechanisms (or different magnetic field configurations) of CMEs in their propagation. The organization of the paper is as follows. In section 2 we describe the numerical simulation setup including the background solar wind and the CMEs initiation mechanisms. We also discuss the energetics of both CMEs. In section 3 we present the comparison between the two models, considering the evolution of the CME-driven shock, CME's sheath and CME's flux rope acceleration. A discussion of the results and conclusions is presented in section 4 .

\section{Numerical Simulation}

[8] An out-of-equilibrium flux rope is superimposed on a steady state background solar wind (SW) generated by the SC component of SWMF. Typically, this component has its domain extend from the low corona $\left(1 R_{\odot}\right)$ to $\sim 24 R_{\odot}$ (for more details on SWMF components see Tóth et al. [2005]). Our steady state SC was simulated using the model presented by Cohen et al. [2007], where the SW is driven by a nonuniform polytropic index distribution [see also Roussev et al., 2003b]. This distribution is used to obtain the heating and the acceleration of the SW plasma and to enable the reproduction of the bimodal SW [Cohen et al., 2008].

[9] Our SC simulation box is limited to a cubic region, that extends from $-12 R_{\odot}<\mathrm{x}, \mathrm{y}, \mathrm{z}<12 R_{\odot}$, centered around the Sun and with the solar rotation axis aligned to the $\mathrm{z}$ axis. The computational domain is divided into blocks, each containing $4 \times 4 \times 4$ cells. The cells range from $3 / 128 R_{\odot}$ on the solar surface out to $3 / 8 R_{\odot}$. For the background $\mathrm{SW}$, we have also resolved the heliospheric current sheet region, in which the blocks were refined to a size $3 / 16 R_{\odot}$.

[10] For the CME propagation the grid was further refined to $3 / 256 R_{\odot}$ within a rectangular box region. The dimensions of this box are $6 R_{\odot} \times 1 R_{\odot} \times 1.8 R_{\odot}(x \times y \times z)$. The box is tilted about the $\mathrm{Y}$ and $\mathrm{Z}$ axis so that it captures the line connecting the center of the Sun and the nose of the CME.

[11] The solar magnetic field is determined from a potential extrapolation of the photospheric magnetic field obtained from the Solar and Heliospheric Observatory (SOHO) /Michelson Doppler Imager (MDI). In our simulation we have chosen Carrington Rotation (CR) 1922 (solar minimum conditions), that represents the period of 27.3 days of Sun's rotation centered on 24 May 1997. This CR1922 was already investigated before by Cohen et al. [2007] and Liu et al. [2008a].

[12] Once the potential coronal magnetic field has been obtained, the SW speed is calculated by the Wang-SheeleyArge (WSA) model [Arge and Pizzo, 2000; Arge et al., 2004]. The WSA model is used together with the Bernoulli integral to determine the polytropic index $(\gamma)$ distribution [Cohen et al., 2007, 2008]. With the $\gamma$ distribution the MHD equations are solved self-consistently. Figure 1 shows the variation of $\gamma$, from $\sim 1.14$ at $2 R_{\odot}$ to $\sim 1.27$ at $6 R_{\odot}$, obtained when generating the steady state $\mathrm{SW}$. The distribution of $\gamma$ "heats" the plasma and produces the required steady state. The amount of heating was estimated by Evans et al. [2009]. The variation of the polytropic index depends on the terminal speed of the SW [Cohen et al., 2008]. Therefore, once the flux rope is inserted and evolved the distribution of $\gamma$ can further vary producing additional heat (that could mimic exchange of energy between the rope and the steady state). In our simulations, to avoid that, we fixed the profile of $\gamma$ after the initialization of the CME. Therefore, we neglect any exchange of energy between the CME and the background solar wind.

[13] To calculate the SW solution, we set up the solar surface temperature to $2 \times 10^{6} \mathrm{~K}$ and density to $5 \times 10^{-16} \mathrm{~g} / \mathrm{cm}^{3}$. After 15,000 steps, a steady state $\mathrm{SW}$ is obtained. This steady state solution reproduces the bimodal SW, with the fast SW at high latitudes and the slow wind at low latitudes. It has the general structure of the coronal magnetic field lines, opened into the heliosphere by the fast wind and closed near the Sun's equator, with a thin current sheet formed along the surface of polarity reversal of the radial magnetic field. The contour plot for the speed of the resulting steady state SW is shown in Figure 2. 


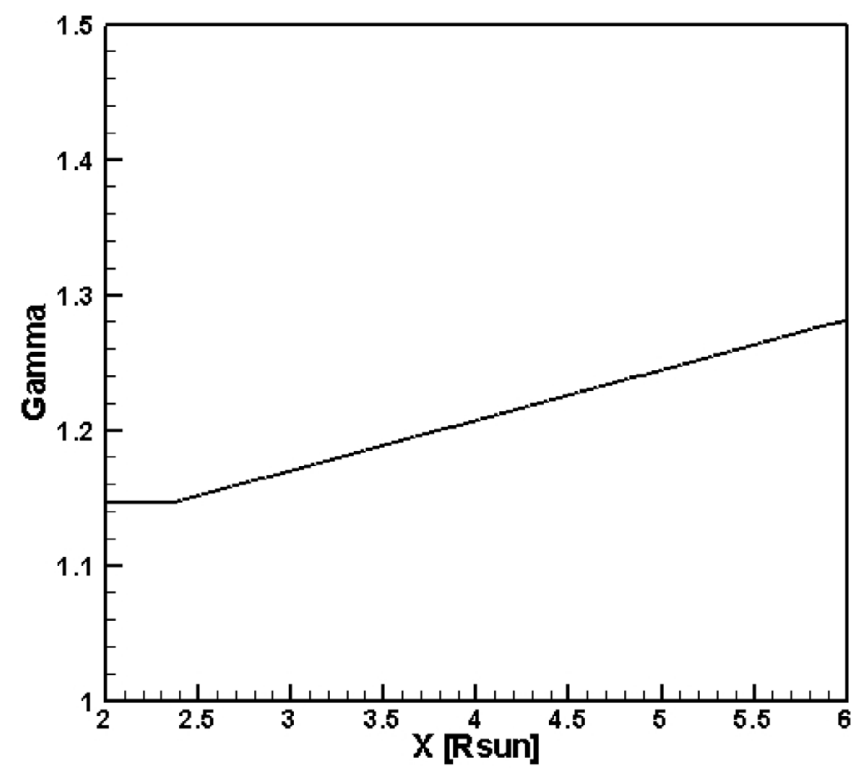

Figure 1. Polytropic index along a line connecting the center of the Sun and the nose of the CME.

\subsection{CME Initialization}

[14] Once the steady state SW is produced, we use GL98 and a modified version of TD99 [e.g., Roussev et al., 2003a; Lugaz et al., 2007; Liu et al., 2008a; Lugaz et al., 2009] to initiate the CMEs and to investigate their signatures while propagating in the lower corona. In the GL98 configuration the field lines have a toroidal configuration. In the modified TD99 the main field is poloidal. So, although both mechanisms are artificial and do not intend to capture all types of CME ejecta we will use them to study the effect of the different magnetic configuration in the CME evolution in the lower corona, beyond $2 R_{\odot}$.

[15] In the GL98 model, a spheromak-type flux rope is distorted into a three-dimensional teardrop shape. Its CME results from an initial force imbalance condition that results from: a removal of part of the plasma in the flux rope and, more significantly, from the insufficient plasma pressure in the background corona to offset the magnetic pressure of the flux rope, which allows the magnetic forces to drive the eruption [Manchester et al., 2004a, 2004b].

[16] For the TD99 model, we use a semicircular flux rope prescribed by a given toroidal current, as in the models by Titov and Démoulin [1999] and Roussev et al. [2003a]. However, we ignored the strapping field produced by the current charges and the magnetic field produced by the infinite line current beneath the photosphere. Consequently, the flux rope solution is superimposed on the ambient magnetic field obtained for the steady state SW, in a situation of nonequilibrium [e.g, Lugaz et al., 2007; Liu et al., 2008a; Lugaz et al., 2009]. Even though this is not a selfconsistent scenario to initiate the CME, the driving force for the eruption is of a magnetic nature. An extra density of $1.7 \times 10^{-17} \mathrm{~g} / \mathrm{cm}^{3}$ is introduced into the flux rope, increasing the inertia without modifying the kinetic pressure and decreasing the internal temperature.

[17] It is important to note that the GL98 and TD99 models were not designed for reproducing the complexity of the flux rope formation and motions at the Sun surface. Our goal is to investigate, once there is an initiation, how the initial magnetic field configuration of the flux ropes could affect their evolution and the shock signatures.

[18] For each initiation mechanism, the flux rope is superimposed within a chosen active region (AR8040) near the solar equator to the same steady state background SW and solar corona solution. Both flux ropes have the same foot point separation and are centered in the same position $\left(x=1.08 R_{\odot}, y=0.27 R_{\odot}, z=0.11 R_{\odot}\right)$. TD99 flux rope has its top portion (toroidal current) aligned with the closed magnetic field lines in the AR8040. The choice for the parallel alignment aims to minimize the transverse drift of the CME. Such flux rope configuration creates a poloidal field almost perpendicular to the field of the active region and is the same as the one used by Liu et al. [2008a]. The same orientation of TD99 was applied to the GL98 flux rope. Figures $3 \mathrm{a}$ and $3 \mathrm{~b}$ show GL98 and TD99 magnetic field lines configuration, respectively, at $t=0 \mathrm{~min}$. The active region and the Sun's magnetic field lines are also shown. Figure 3a shows that GL98 toroidal field lines are parallel to the active region field and perpendicular to the coronal field, while Figure $3 \mathrm{~b}$ shows that the TD99 poloidal field is perpendicular to the active region field and antiparallel to the coronal field.

[19] The initial TD99 flux rope was generated with a current $I=5 \times 10^{11} \mathrm{~A}$, as in work by Liu et al. [2008a]. The flux rope was set with a major radius $\left(R_{f}\right)$ of $0.14 R_{\odot}$, a minor radius $a=0.026 R_{\odot}$, and the center of the magnetic torus below the Sun's surface at $d=25 \mathrm{Mm}$ or $0.035 R_{\odot}$. The GL98 initial flux rope was specified by setting $a=0.7 R_{\odot}$, $r_{0}=0.75 R_{\odot}, r_{1}=1.8 R_{\odot}, a_{1}=0.93$, where $a$ is the stretching length of the flux rope into a teardrop shape in the radial direction, $r_{0}$ is the radius of the spherical flux rope, $r_{1}$ is the distance from the solar center where the spherical flux rope is placed (prior to the radial contraction) and $a_{1}$ is a free parameter that determines the magnetic field strength and plasma pressure in the flux rope. The GL98 parameters

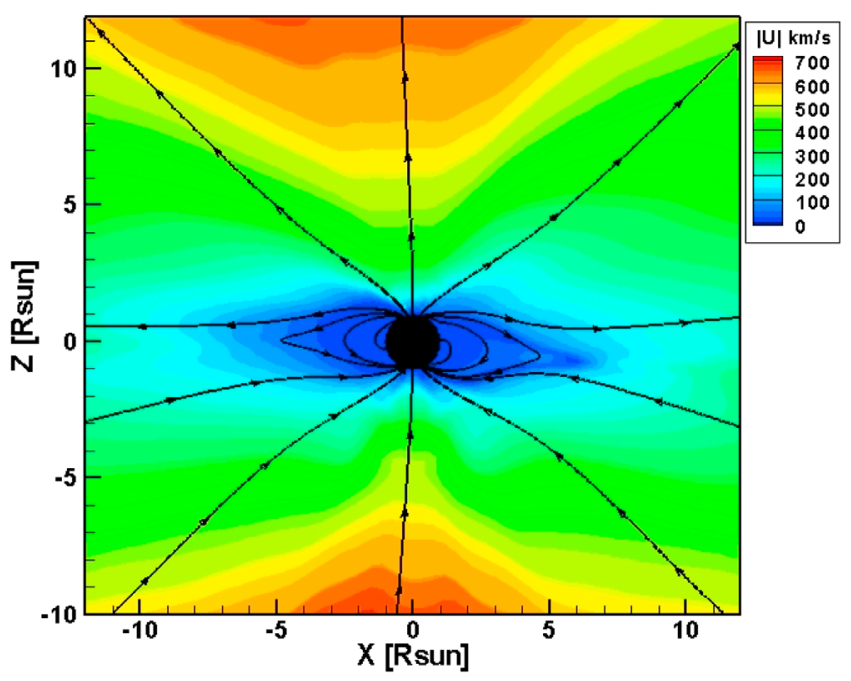

Figure 2. Contour plot for the speed of the steady state solar wind solution for CR1922 in the x-z plane. The solid black lines are the magnetic field lines. 
(a)

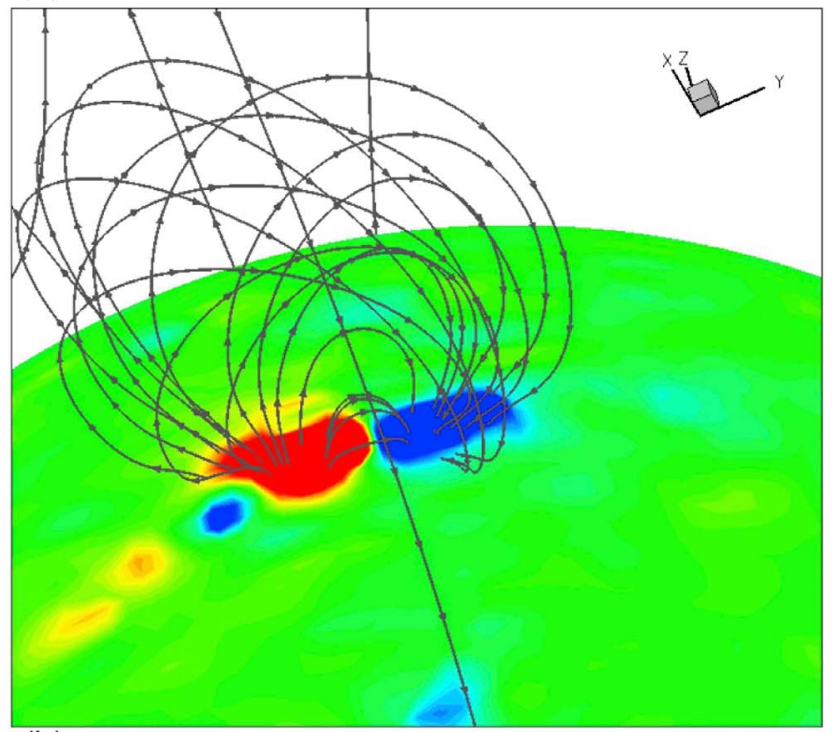

(b)

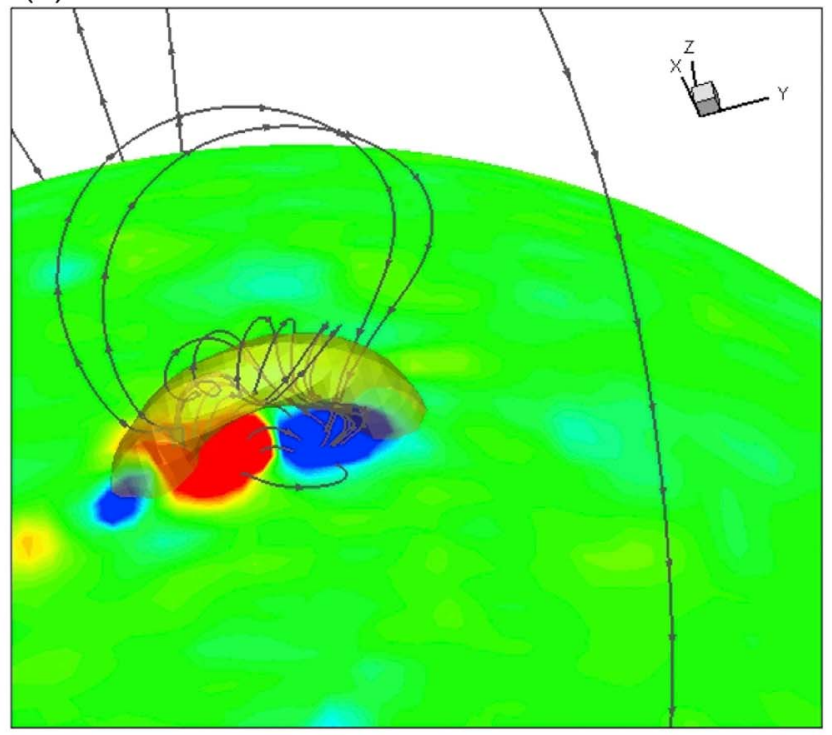

Figure 3. Configuration of the magnetic field lines for both flux ropes, (a) GL98 and (b) TD99, the active region, and the Sun.

were selected in order to have a GL98 flux rope within the same foot point separation as the one defined for the TD99 flux rope. Such choice of parameters provides a GL98 flux rope with a larger structure and height than the TD99 flux rope. However, both flux ropes initial density has the same order of magnitude $\left(\sim 10^{-16} \mathrm{~g} / \mathrm{cm}^{3}\right)$. Within this configuration, we obtained two different CMEs erupting in the same background steady state.

\subsection{CME Energetics}

[20] Both GL98 and TD99 configurations guaranteed that their CMEs begin rising right after the flux ropes were inserted, despite their topological differences. Here, we analyze the energetics of both CMEs during their eruption and evolution as a function of distance. In our analysis, we estimated the energy at a certain time (or position) using the whole simulation box and then subtracted the energy calculated from the steady state solution.

[21] The introduction of the GL98 flux rope to the corona added $4.7 \times 10^{16} \mathrm{~g}$ of mass into the solar corona and an average magnetic field strength of $1.5 \mathrm{G}$. Such configuration
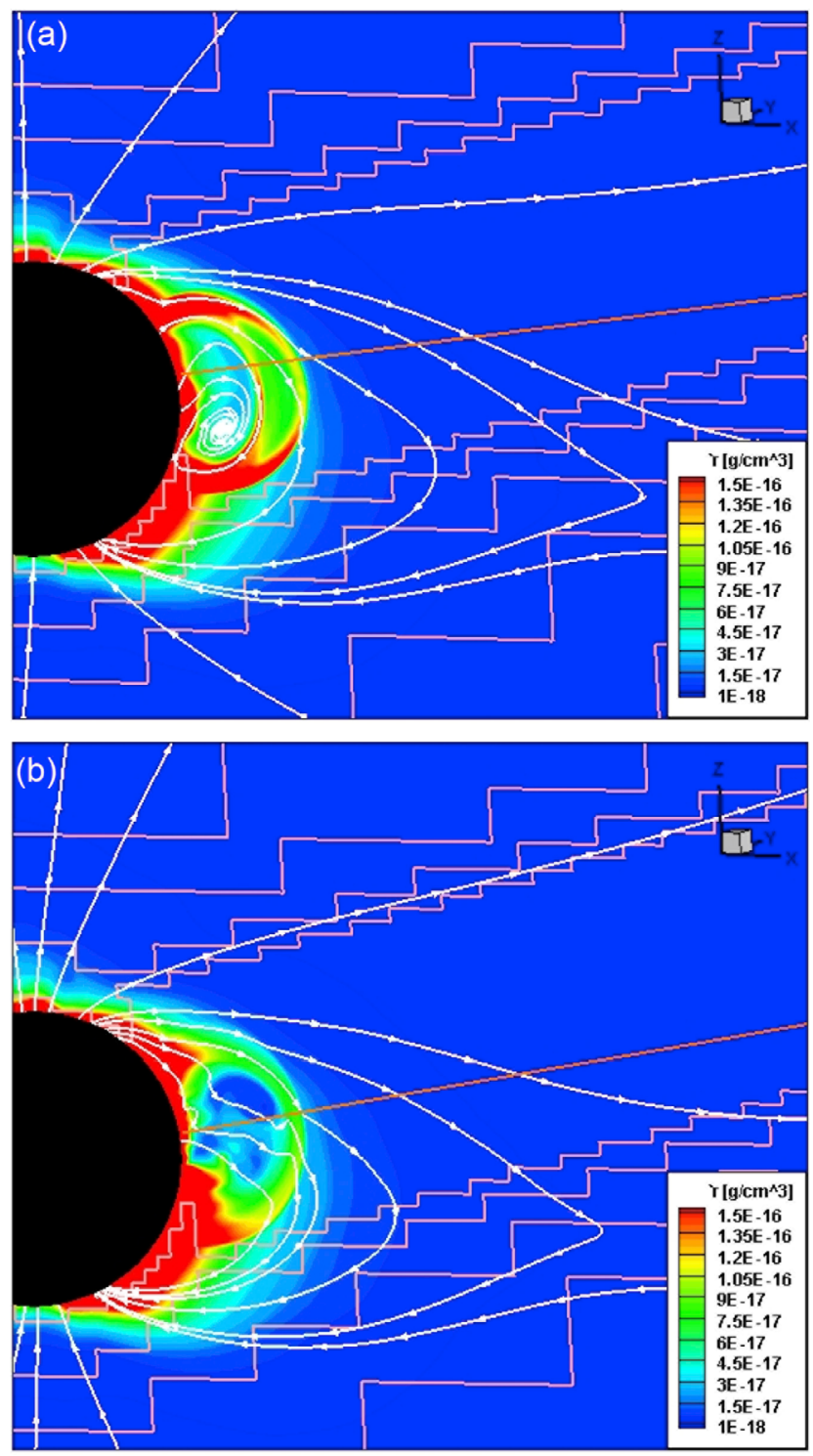

Figure 4. Density contour plot on the lower corona when the CME-driven shock is at $2 R_{\odot}$ on a meridional slice intersecting the nose of the CME for (a) GL98 and (b) TD99. The pink lines limit the regions with different refinement levels, and the white lines represent direction of the magnetic field lines confined to the 2-D plane (the magnetic field lines are integrated on the plane ignoring the component out of the plane). The orange line indicates the lines that go through the nose of the CME for each case. This line is used to locally explore the evolution of the CME shock and sheath region as reported in Figures 6, 7, 9, 10, and 12. GL98 and TD99 ( $t>10 \mathrm{~min})$ were not extracted at the same line and so were not their correspondent background since the GL98 CME nose was $1^{\circ}-2^{\circ}$ of latitude above the TD99 CME nose. 

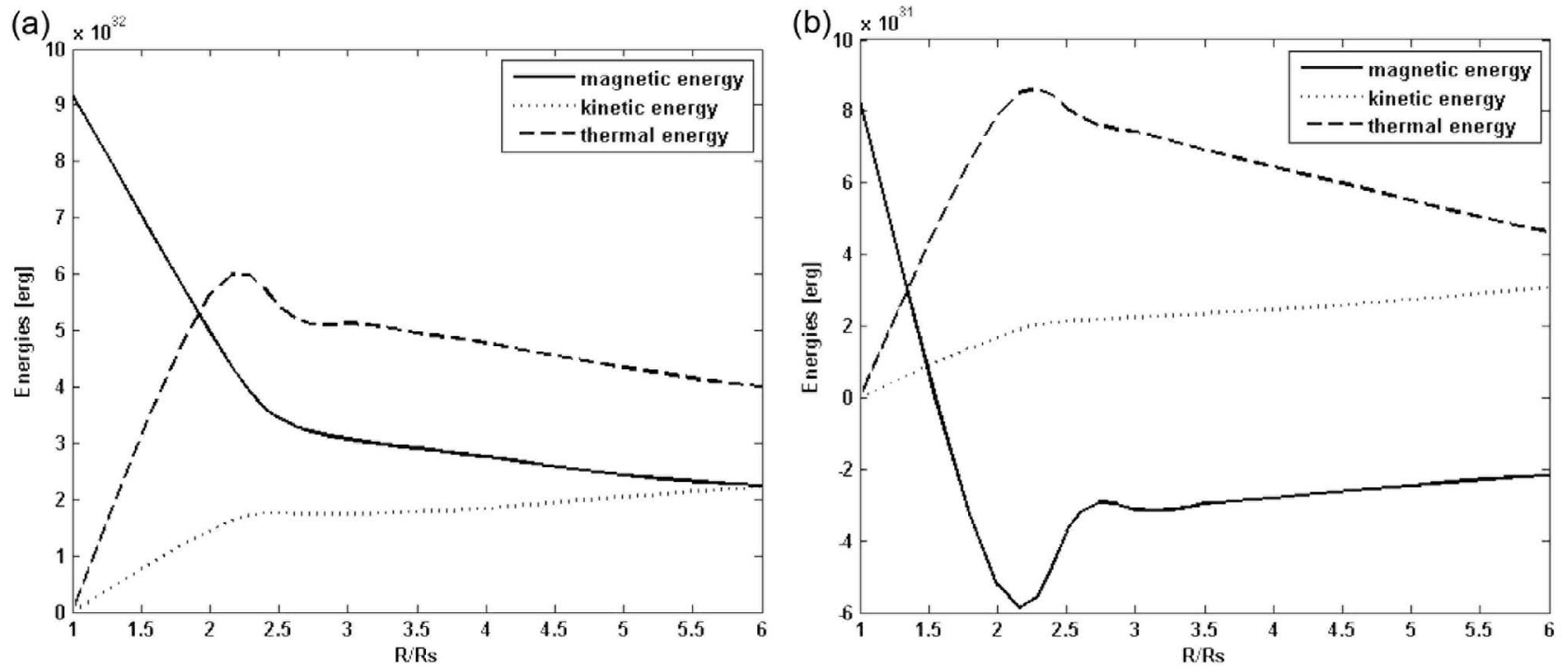

Figure 5. Energy profile for both CMEs, (a) GL98 and (b) TD99, until $6 R_{\odot}$. The energy at each position is computed using the whole simulation box, subtracting the corresponding energy calculated from the steady state solution.

has increased the coronal energy on $9.0 \times 10^{32}$ ergs of magnetic energy (and negligible thermal energy). In the GL98 model the added plasma is concentrated in a prominence-type core at the base of the flux rope. The TD99 flux rope, on the other hand, presents a stronger average magnetic field strength $5 \mathrm{G}$ and adds $1.4 \times 10^{12} \mathrm{~g}$ of mass to the corona. The TD99 configuration increased the coronal magnetic energy on $8.0 \times 10^{31}$ ergs (and negligible thermal energy). In the TD99 flux rope the added plasma was equally distributed along the flux rope. Despite their differences, when the flux ropes are inserted onto the active region the increase on the photospheric magnetic field is less than $1 \%$. Our results indicate that both CMEs are initially driven by the added magnetic energy.

[22] Right in the early stages of the CMEs evolution, the formation of a shock can be observed ahead of them. Figure 4 shows the refined grid around the CME-driven shock when it is at $2 R_{\odot}$ on a meridional slice intersecting the nose of the CME, for GL98 (Figure 4a) and TD99 (Figure 4b). The differences observed in the magnetic field lines are due to the fact that the toroidal component of the magnetic field is stronger for GL98 and the poloidal is stronger for TD99. Both CMEs were tracked until $6 R_{\odot}$ and the effect of both driving mechanisms on their evolution will be presented in section 3 .

[23] When the CME-driven shock is at $2 R_{\odot}$ the GL98 $\mathrm{CME}$ presents $5.6 \times 10^{32}$ ergs of thermal energy, $1.5 \times$ $10^{32}$ ergs of kinetic energy and $5 \times 10^{32}$ ergs of magnetic energy. The total initial magnetic energy gets transferred not only to the kinetic energy but a larger portion to the thermal energy. At $2 R_{\odot}$, the TD99 CME increased the background steady state thermal energy by $7.8 \times 10^{31} \mathrm{ergs}$, the kinetic energy on $1.8 \times 10^{31}$ ergs and the magnetic energy on $-5.3 \times 10^{31}$ ergs. Again, the total initial magnetic energy gets transferred not only to the kinetic energy but a larger portion to the thermal energy. We observe that, at this distance from the Sun, the TD99 CME magnetic energy is negative. This result is due to magnetic dissipation (reconnection). The numerical reconnection in the code is inherent to any finite difference scheme. TD99 configuration has a more twisted magnetic field configuration (see Figure 3b). Its poloidal component is perpendicular to the active region field that favors its reconnection. GL98 has only a toroidal component and its oriented parallel to the active region field. However, the TD99 poloidal field is antiparallel to the large coronal field, while the GL98 toroidal field is perpendicular. Therefore we expect more reconnection to occur for the TD99 model. It is important to mention that though we have a very high grid resolution, it is questionable whether or not the degree of magnetic dissipation of the TD99 flux rope is realistic, great effort was made to limit magnetic dissipation, but it may still be larger than what is expected in reality.

[24] Figures 5a and 5b show GL98 and TD99 CMEs energy profiles in the lower corona, respectively. We observe that for both CMEs the thermal energy is dominant when compared to the kinetic and magnetic energy energies. Manchester et al. [2004b] suggested that the initial magnetic energy is converted into thermal energy for a GL98 CME. They argue that the thermal energy is absorbed by coronal heat sources and, consequently, exhibits only a short-lived increase before falling slightly below the initial value and the CMEs kinetic energy. Our results do show that the magnetic energy gets converted into thermal energy in agreement with their results. However, our thermal energy is much larger than the kinetic energy until $6 R \odot$. One of the reasons to such difference in the results, may be due to the different thermodynamics properties of the solar wind models. Another reason could be the pilling up of hot material from the much hotter background. Manchester et al. [2004b] assume an ideal gas with a $\gamma=5 / 3$ while in our simulations $\gamma<5 / 3$ (as shown in Figure 1) and the solar wind is heated as it propagates away from the Sun. Additionally, our heating source is fixed (by $\gamma$ ) and does not vary in space as in their simulation. This configuration is 

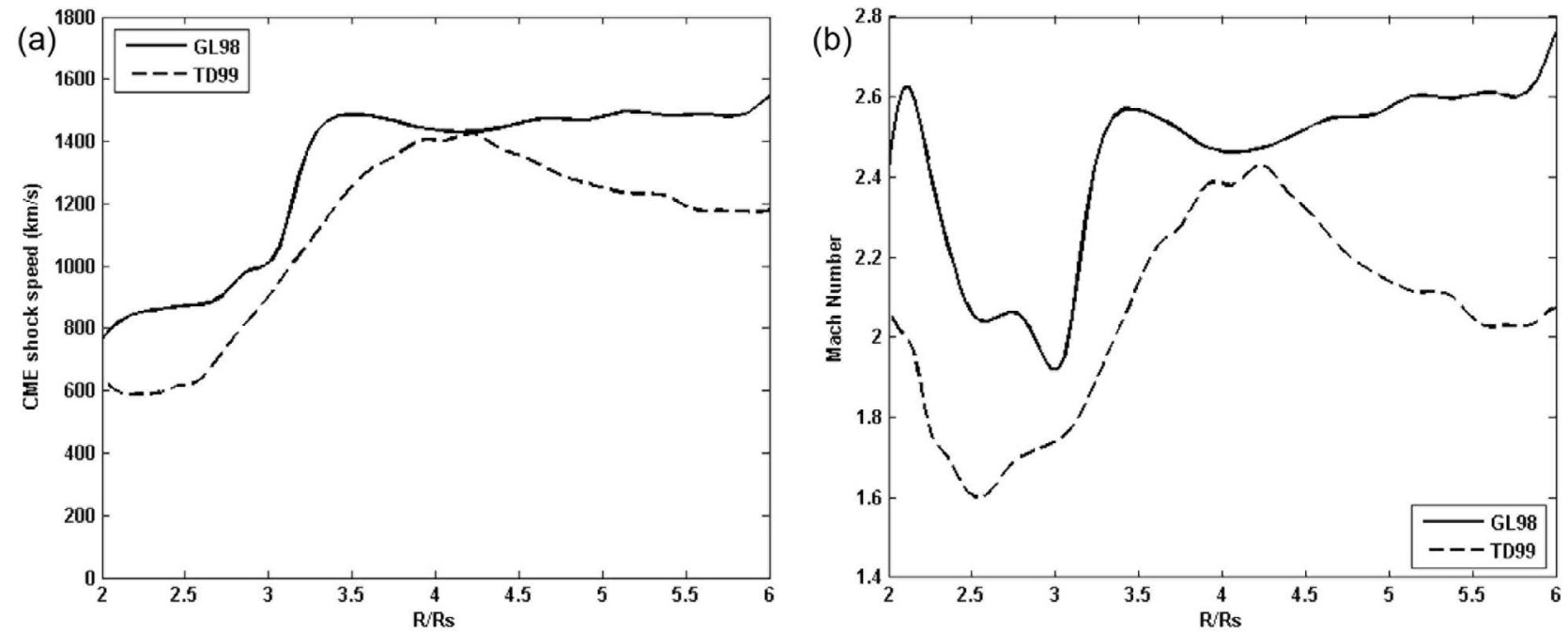

Figure 6. (a) Comparison between GL98 (solid line) and TD99 (dashed line) models in terms of their shock speed. (b) Fast Mach number comparison in the upstream frame (see equation (2)) for the GL98 (solid line) and TD99 (dashed line) models versus the shock position.

affecting the CMEs evolution and the thermal energy starts to dominate the magnetic energy after $2 R_{\odot}$. These results indicate that the background solar wind plays an important role in the CMEs evolution.

[25] The remaining kinetic energies for the two cases differ due to the difference in mass between the two CMEs. An interesting result is that, although the kinetic energy of the GL98 CME is an order of magnitude larger than the TD99 CME at $2 R_{\odot}$, the velocity of the shocks, as shown in Figure 6a (section 3), are almost identical. The difference is due to the mass carried by the GL98 CME that is larger than the mass carried by the TD99 CME (at $2 R_{\odot}$ the $\mathrm{CME}$ mass for GL98 is $5 \times 10^{16} \mathrm{~g}$ while the CME mass for TD99 is $\left.1.8 \times 10^{14} \mathrm{~g}\right)$.

\section{Evolution of the CMEs in the Lower Corona}

[26] To explore the evolution of both CMEs in the lower corona, we have defined a line going from the center of the Sun to the CME's nose. Along this line we compare the variations of plasma density, velocity, magnetic field, pressure and acceleration for both CMEs. We also compare the behavior of their driven shocks.

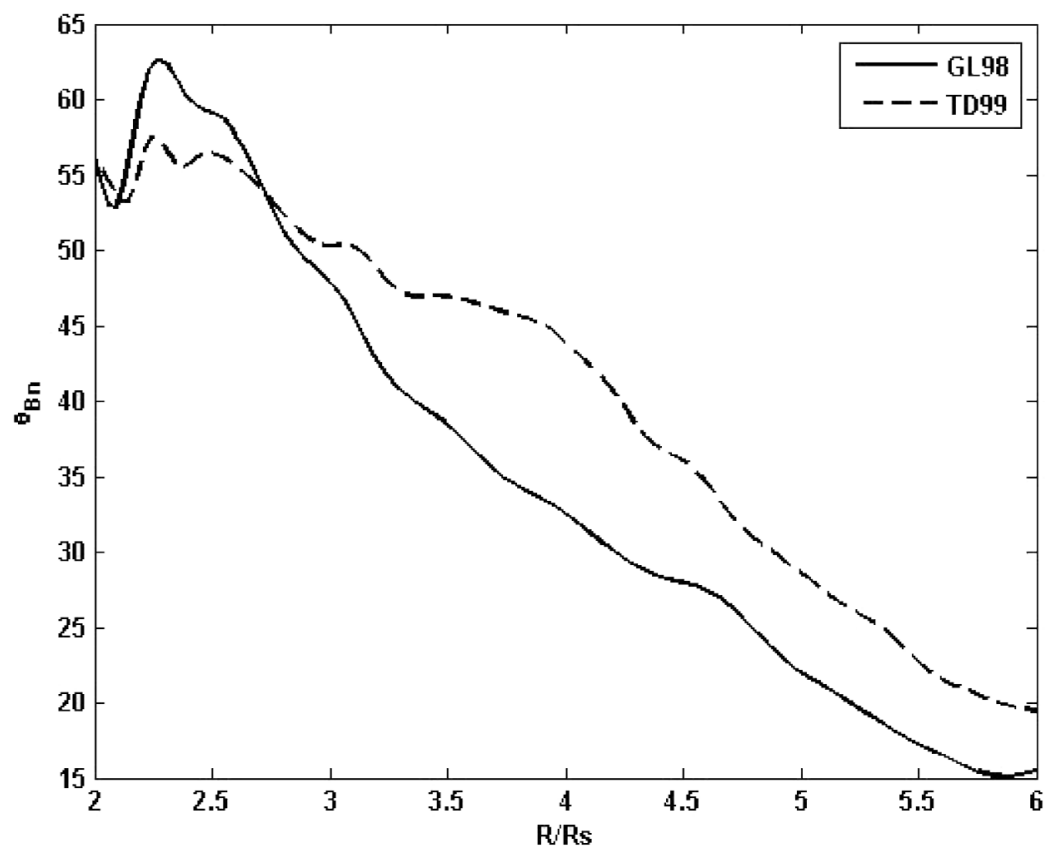

Figure 7. Comparison of the angle between the shock normal and the upstream magnetic field $\left(\theta_{B n}\right)$ for GL98 (solid line) and TD99 (dashed line). 

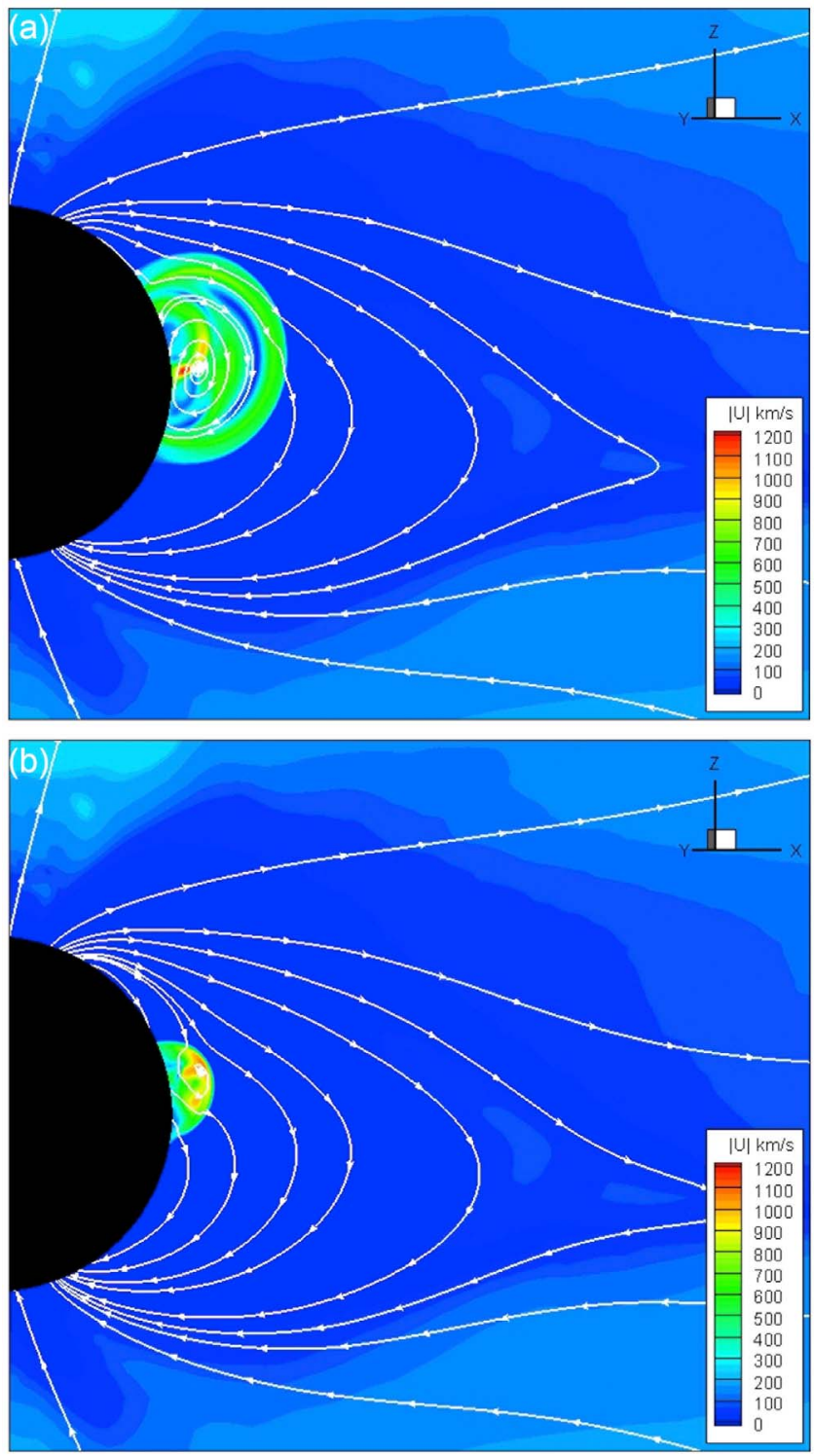

Figure 8. Velocity contour plot on the lower corona at $t=$ $2 \mathrm{~min}$ on a meridional slice intersecting the nose of the CME for (a) GL98 and (b) TD99. The white lines represent direction of the magnetic field lines confined to the 2-D plane (the magnetic field lines are integrated on the plane ignoring the component out of the plane).

\subsection{CME-Driven Shocks Comparison}

[27] Understanding CME-driven shocks and their role in particle acceleration have become one of space weather goals [e.g., Le and Han, 2005; Manchester et al., 2005]. A crucial part of it includes understanding how different driving CME processes would affect the shock evolution characteristics. We have tracked the driven shocks within the GL98 and TD99 models as they propagate in the lower corona, until $6 R_{\odot}$. We set the highest resolution through the front part of the CME (the nose) and analyze the mechanism signatures only at the nose of the CME. The evolution of the shock is followed by the shock speed, shock compression ratio, Mach number $\left(\mathrm{M}_{\mathrm{A}}\right)$ and $\theta_{B n}$, the angle between the shock normal and the upstream magnetic field.

[28] The shock speed was calculated using the RankineHugoniot relation

$$
V_{s}=\frac{\rho_{u p} \mathbf{U}_{u p} \cdot \mathbf{n}-\rho_{\text {down }} \mathbf{U}_{\text {down }} \cdot \mathbf{n}}{\rho_{u p}-\rho_{\text {down }}},
$$

where $\rho_{u p}($ down $)$ and $\mathbf{U}_{u p}($ down $)$ represent the density and the velocity upstream (downstream) of the shock, respectively, and $\mathbf{n}$ is the shock normal [e.g., Lugaz et al., 2007; Liu et al., 2008a]. In equation (1), the shock normal $\mathbf{n}$ was calculated using the velocity coplanarity relation: $\mathbf{n}=$ $\left(\mathbf{U}_{u p}-\mathbf{U}_{\text {down }}\right) /\left(\left\|\mathbf{U}_{u p}-\mathbf{U}_{\text {down }}\right\|\right)$ [e.g., Kivelson and Russel, 1995]; $\mathbf{n}$ can also be obtained as a function of the magnetic field ( $\mathbf{B}_{u p}$ and $\mathbf{B}_{\text {down }}$ ). We chose to use velocity to avoid the high variability of magnetic field downstream in the simulation.

[29] Our results show that despite the CMEs different initial magnetic field configuration and magnetic energy, both CMEs present very similar shock speeds. Figure 6a shows the shock speed for the GL98 (solid line) and TD99 (dashed line) models.

[30] Both CMEs shock speeds, GL98 and TD99, are faster than the background solar wind, that at the streamer is few $\mathrm{km} / \mathrm{s}$. The shock speeds increase around $R=3 R_{\odot}$ because within 3-3.5 $R_{\odot}$ both GL98 and TD99 CME-driven shocks are still within the closed loops of the streamer. After 3-3.5 $R_{\odot}$, the shocks change their orientation and leave the region of the streamer with closed loops and accelerate. For the GL98 model, the CME shock speed ranges from $\sim 780 \mathrm{~km} / \mathrm{s}$ (at $2 R_{\odot}$ ) to $\sim 1550 \mathrm{~km} / \mathrm{s}$ (at $6 R_{\odot}$ ), while for the TD99 model it ranges from $\sim 630 \mathrm{~km} / \mathrm{s}$ (at $2 R_{\odot}$ ) to $\sim 1180 \mathrm{~km} / \mathrm{s}$ (at $6 R_{\odot}$ ). Our results are in agreement with previous simulations and observations results presented in the literature, with shock speeds ranging $\sim 300 \mathrm{~km} / \mathrm{s}$ and $\sim 1500 \mathrm{~km} / \mathrm{s}$ from the lower corona to the interplanetary medium [e.g., Sheeley et al., 1985; Cane et al., 1987; Watari and Detman, 1998; Mancuso and Bemporad, 2009]. Roussev et al. [2004], for example, presented a shock speed of $\sim 1200 \mathrm{~km} / \mathrm{s}$ at $4 R_{\odot}$, and Manchester et al. [2005] found a shock speed of $\sim 850 \mathrm{~km} / \mathrm{s}$ at $8 R_{\odot}$ for a CME initiated by the GL98 model.

[31] The slightly higher shock speed for the GL98 CME is due to the higher magnetic field in its flux rope. This result is in agreement with the analysis presented by Manchester et al. [2008]. Manchester et al. [2008] presented a simulation of two of the Halloween CMEs, CME1 (26 October 2003) and CME2 (28 October 2003), as observed by LASCO, using the TD99 model. They observed that CME2, which had a stronger magnetic field, would drive a faster shock ahead of it then CME1. Their results showed that CME1-driven shock took $\sim 20 \mathrm{~min}$ to get to $5.5 R_{\odot}$ with a speed of $\sim 2100 \mathrm{~km} / \mathrm{s}$, and $\sim 1.7 \mathrm{~h}$ to get to $20 R_{\odot}$ with a speed of $1500 \mathrm{~km} / \mathrm{s}$; while CME2-driven shock took $\sim 15 \mathrm{~min}$ to get to $9 R_{\odot}$ with a speed of $3200 \mathrm{~km} / \mathrm{s}$ and less than $1 \mathrm{~h}$ to get to $20 R_{\odot}$ with a speed of $2800 \mathrm{~km} / \mathrm{s}$. Our results show that the GL98 CME drives slightly faster shock ahead of it than the TD99 CME, with the GL98 CME-driven shock getting to $5 R_{\odot}$ with a speed of $\sim 1500 \mathrm{~km} / \mathrm{s}$ in $\sim 35 \mathrm{~min}$ and the 

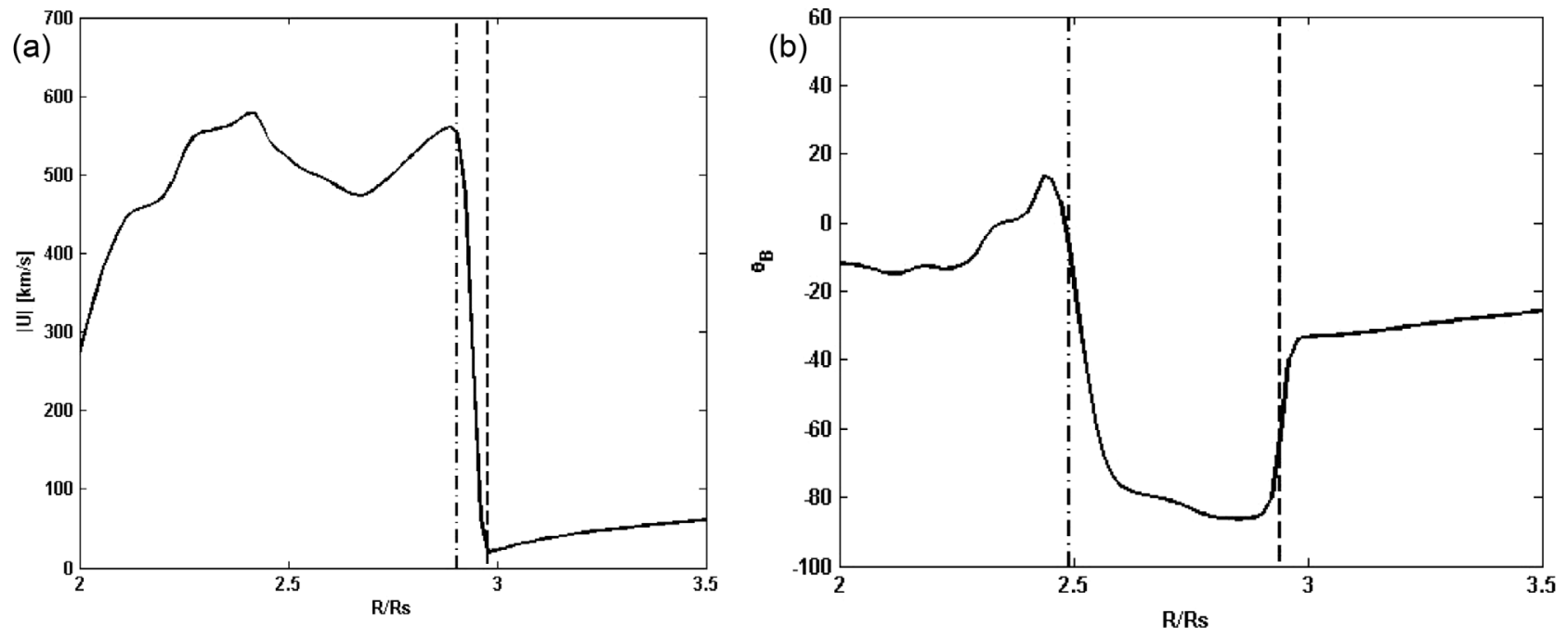

Figure 9. CME shock and sheath regions. (a) Shock region obtained from the velocity profile, defined as the mean point between the dashed and the dash-dotted lines. (b) Latitude of the magnetic field vector $\left(\theta_{B}\right)$ profile with the sheath upstream (dashed line) and downstream (dash-dotted line) indicated. (Profiles for TD99 model at $t=26 \mathrm{~min}$ ).

TD99 CME-driven shock getting to $5 R_{\odot}$ in $\sim 52$ min with a speed of $\sim 1250 \mathrm{~km} / \mathrm{s}$.

[32] We also analyzed the evolution of the fast Mach number $\left(\mathrm{M}_{\mathrm{A}}\right)$ in the upstream frame,

$$
M_{A}=\frac{\left(V_{s}-\left(\mathbf{U}_{u p} \cdot \mathbf{n}\right)\right)}{\sqrt{\left(V_{A}^{2}+V_{c}^{2}\right)}},
$$

where $V_{s}$ is the shock speed, $\mathbf{U}_{u p}$ represents the velocity upstream of the shock, $\mathbf{n}$ is the shock normal and $V_{A}$ and $V_{c}$ are the Alfvén speed and the sound speed, respectively. Our results show that, as observed for the shock speed, the general profile of $\mathrm{M}_{\mathrm{A}}$ in the lower corona for both models is very similar, despite their different magnetic initial configuration and different magnetic energy. We observe, in Figure $6 \mathrm{~b}$, that $\mathrm{M}_{\mathrm{A}}$ tends to increase as the shock travels through the slow solar wind, as expected. $\mathrm{M}_{\mathrm{A}}$ increases up to $\sim 2.8$ at $6 R_{\odot}$ for the GL98 model, while for the TD99 model it increases up to $\sim 2.1$ at $6 R_{\odot}$. Manchester et al. [2005] observed the same behavior for a CME initiated by the GL98 model between 8 and $16 R_{\odot}$. In their work the CME presents a $\mathrm{M}_{\mathrm{A}} \sim 2.5$ at $8 R_{\odot}$ increasing up to $\sim 4.5$ at $16 R_{\odot}$. Our present work shows that the same behavior occurs in the lower corona. Manchester et al. [2005] also discussed a decrease in $\mathrm{M}_{\mathrm{A}}$ as the shock propagates further out into the interplanetary medium, for $R>16 R_{\odot}$ for the GL98 CME. In our results in Figure $6 \mathrm{~b}$ we observe a decrease in $\mathrm{M}_{\mathrm{A}}$ for the TD99 CME for $R>4 R_{\odot}$, coinciding with the deceleration of the shock (shown in Figure 6a). While the CME propagates material is swept up by it and deceleration happens due to momentum conservation. However, the fact that the magnetic energy dissipates quickly could also play a role decelerating the shock. As discussed before, TD99 CME magnetic energy dissipates due to numerical reconnection that is favored by its more twisted magnetic field configuration. Our results also indicate that the GL98 model would drive shocks more likely to present accelerated particles in the lower corona due to its higher shock compression. The higher shock compression in the GL98 CME is, in this case, probably due to its higher magnetic energy and higher thermal energy (due to sweeping of the solar wind) and, also, to its more rapid expansion. The difference between GL98 and TD99 shock compression can also be related to the fact that the GL98 CME has a faster shock speed and higher Mach number than the TD99 CME.

[33] Mancuso and Bemporad [2009] analyzed two fast CME-driven shocks observed with the UltraViolet Coronagraph Spectrometer (UVCS) on board the Solar and Heliospheric Observatory (SOHO), in the lower corona. For a CME observed on 22 March 2002 they observed a shock speed ranging from $\sim 100 \mathrm{~km} / \mathrm{s}$ to $\sim 1700 \mathrm{~km} / \mathrm{s}$ at $\sim 4 R_{\odot}$ and Mach numbers between 1.5 and 2.4. For a CME observed on 23 March 2002, they found Mach numbers between 1.5 and 2.1. Both CME-driven shocks also presented compression ratios of $\sim 2.2$ to $\sim 2.4$. Our results for the GL98 and the TD99 CMEs are comparable, in the lower corona, to the results presented by Mancuso and Bemporad [2009].

[34] An important result from our simulations is shown at the analysis of the angle between the shock normal and the upstream magnetic field $\left(\theta_{B n}\right)$. Figure 7 presents the results we have obtained for $\theta_{B n}$ at the CME-driven shocks using the GL98 and TD99 models. The results show the shocks are quasi-parallel at the nose of the $\mathrm{CME}\left(R>3-3.5 R_{\odot}\right)$ and that there is a decrease of $\theta_{B n}$ while the CME-driven shock evolves until $6 R_{\odot}$. However, in the very early stages of both CMEs evolution $\left(R<3-3.5 R_{\odot}\right)$, we observe that the shock is quasi-perpendicular at the nose, indicating that particles can be more efficiently accelerated in the lower corona. That occurs because, in earlier times, the CME is within the closed loops of the streamer that have not opened up yet, as we can see in Figure 8. Manchester et al. [2005] analyzed $\theta_{B n}$ at a line going through the nose of a CME initiated using the GL98 model. Their results show that the shock is quasi-parallel at the nose of the CME for $R>8 R_{\odot}$. They observed that for $8 R_{\odot}<R<20 R_{\odot}, \theta_{B n}$ is between $0^{\circ}$ and $15^{\circ}$ (as shown in their Figure 10a). Our results show an 

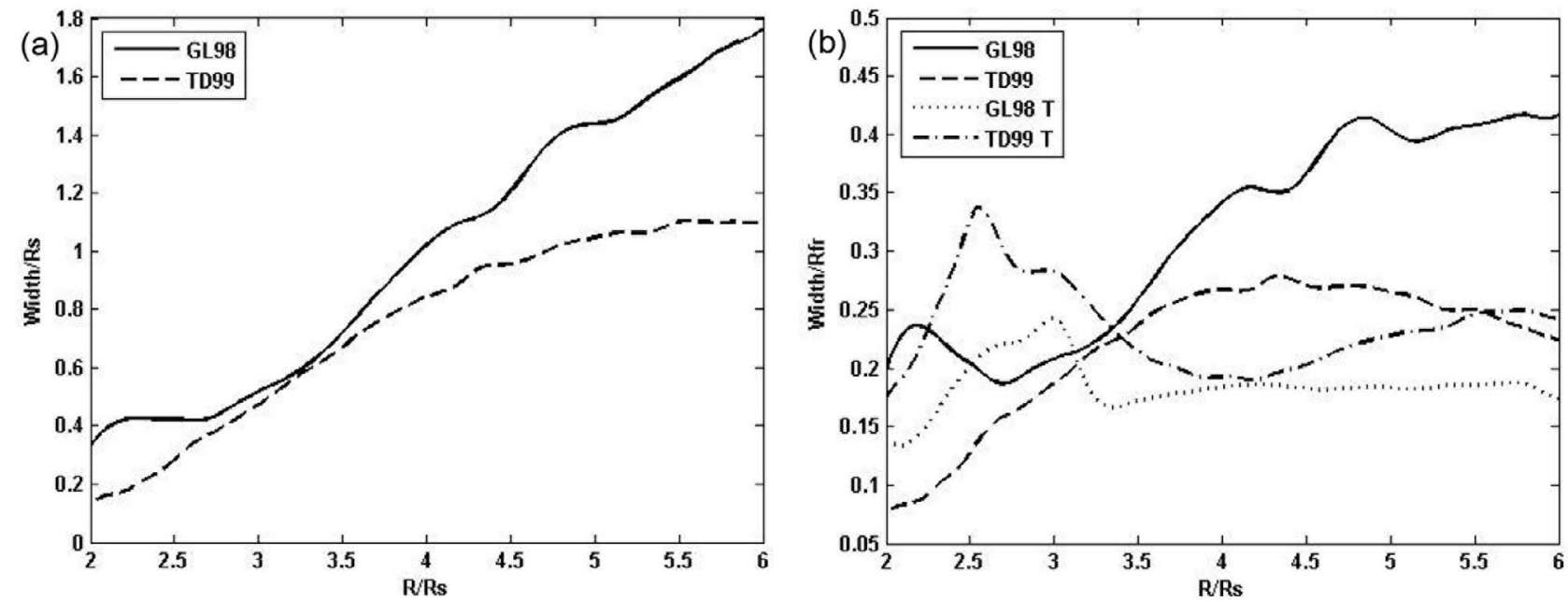

Figure 10. (a) Evolution of the width of the CME sheath normalized by $R_{\odot}$ for both driving mechanisms: GL98 (solid line) and TD99 (dashed line). (b) CME sheath width evolution normalized by $R_{f r}$. The dotted and dash-dotted lines show the theoretical results for the CME sheath width for GL98 and TD99, estimated by width $/ R_{f r}=(0.8 / \eta)$ [e.g., Siscoe and Odstrcil, 2008].

agreement with that for $R>5.5 R_{\odot}$. At $6 R_{\odot}$, for example, $\theta_{B n} \sim 15.4^{\circ}$ for the GL98 model and shows a tendency of $\theta_{B n}<15^{\circ}$ after that, and for the TD99 model $\theta_{B n} \sim 19.4^{\circ}$. It is important to mention here that our analyzes is restricted to a region close to the CME nose. Behavior at the flanks can be very different and should be a topic of future investigations.

[35] These results indicate that differences in CMEs initial configuration, such as their magnetic field configuration and magnetic energy, are not distinguishable by their shock speeds, Mach number and $\theta_{B n}$ profiles.

\subsection{CMEs: Sheath and Postshock Compression}

[36] Here we investigate the signatures of the CMEs sheath in the lower corona, for both models. As these CME flux ropes propagate away from the Sun, a dense plasma is pushed ahead of them, a result of the CME-driven shock. Right behind the shock, between the shock front and the $\mathrm{CME}$, there is a region of shocked solar wind called the CME's sheath. Observations at $1 \mathrm{AU}$ have shown that the sheath region can be as geoeffective as the CME themselves [Tsurutani et al., 1988] and that they have an important implication on the generation of geomagnetic storms [ Liu et al., 2008b]. A CME's sheath can bring up to $29 \%$ additional energy into the Earth's magnetosphere during geomagnetic storms [Zhang et al., 2008]. Additionally, the turbulence in the CME sheath, following the shock, is an important ingredient in the acceleration process of energetic particles [Liu et al., 2008b].

[37] To perform our analysis we have considered the shock position as the average position between the dashed line and dash-dotted line limits on the velocity profile, as indicated in Figure 9a. The finite shock width (between the lines in Figure 9a) is due to numerical resolution. We define the sheath as the region between the shock and the flux rope. The sheath upstream limit is defined as the shock position, represented by the dashed line in Figure 9b. The downstream of the sheath is defined as a sharp change in the angle $\theta_{B}$, following the work of Burlaga [1988] where he defined
$\theta_{B}=\sin ^{-1} \frac{B_{n}}{B}$, where $B_{n}$ is the direction normal to the ecliptic plane and $B$ is the magnetic field strength. He showed that sharp changes in this angle marked the edges of a flux rope lying in the ecliptic plane and whose axis was perpendicular to the Sun-Earth line. Evans et al. [2011] have used such angle to identify the region separating the shocked solar wind from the ejecta. The dash-dotted line in Figure $9 \mathrm{~b}$ represents the downstream of the sheath.

[38] CMEs expand with the distance from the Sun and CMEs sheaths increase in size as well. Figures 10a and 10b show the evolution of the CME sheath width normalized by $R_{\odot}$ for the GL98 (solid line) and TD99 (dashed line) models. We observe in Figure 10a that the expansion rate of the sheath width is similar for both models. The CME initialized with the GL98 model presents a wider sheath than with TD99 all the way to $6 R_{\odot}$. At $6 R_{\odot}$, for the GL98 model, the CME sheath width $\left(1.8 R_{\odot}\right)$ is almost double the TD99 CME sheath width $\left(\sim 1.1 R_{\odot}\right)$. Figures $11 \mathrm{a}$ and $11 \mathrm{~b}$ illustrate both GL98 and TD99 CMEs sheath width, respectively, when the CME-driven shock is at $6 R_{\odot}$. Figures $11 \mathrm{a}$ and $11 \mathrm{~b}$ present a contour plot of $\theta_{B}$ on a meridional slice intersecting the nose of the CME. The blue contour indicates the sheath of the CME and the white lines represent the magnetic field lines.

[39] Siscoe and Odstrcil [2008] consider sheaths that are formed around solar system objects as propagation sheath or expansion sheath. When the solar wind enters the sheath at a shock wave, which wraps around the object, its magnetized plasma is deflected sideways, at the nose, so as to flow around the object through a more or less steady state flow region (the sheath itself), then leaves the object behind. These sheaths are called propagation sheaths. By contrast, the term expansion sheath is used to refer to a sheath that forms around an object that expands but does not propagate relative to the solar wind. In this case, the solar wind piles up all around the object. For a fast, pure expansion sheath, the shock stands off from the expanding body a distance that is less than for a pure propagation sheath with matched speed and radius of curvature of the body. The authors also 
(a)

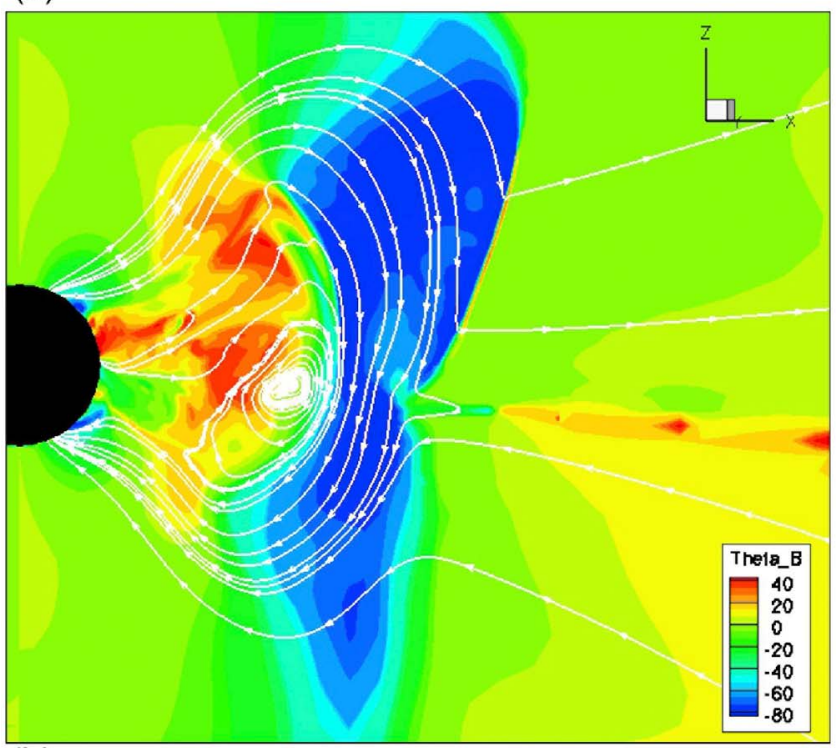

(b)

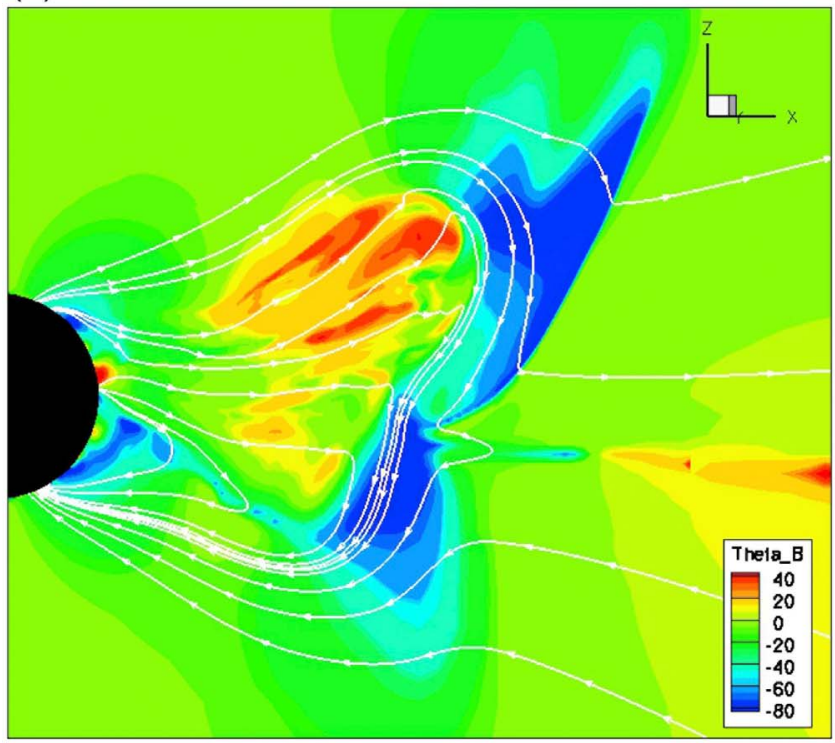

Figure 11. $\theta_{B}$ contour plot on the lower corona when the CME-driven shock is at $6 R_{\odot}$ on a meridional slice intersecting the nose of the CME for (a) GL98 and (b) TD99. The blue contour indicates the sheath of the CMEs, and the white lines represent direction of the magnetic field lines confined to the 2-D plane (the magnetic field lines are integrated on the plane ignoring the component out of the plane).

present an expression for the CME sheath width normalized by the radius of curvature of the flux rope $\left(W / R_{f r}\right)$ at the nose of the shock: $W / R_{f r}=0.8 / \eta$, where $\eta$ is the postshock to preshock ratio of the mass densities and is a function of $\gamma$, shock and solar wind velocities (e.g., Appendix A of Siscoe and Odstrcil [2008]). They argue that a pure propagation sheath has $W / R_{f r} \geq 0.2$ while for a pure expansion sheath $W / R_{f r} \sim 0.1$. They also suggested that CMEs sheaths can present characteristics of both, pure propagation and pure expansion sheaths. Assuming that the CMEs propagate spherically, we calculated the value of $W / R_{f r}$ for both models we use here. Results for GL98 and TD99 are presented in Figure $10 \mathrm{~b}$.

[40] Our results in Figure 10b show that the TD99 CME sheath width is comparable to its theoretical prediction all the way to $6 R_{\odot}$. However, the GL98 CME sheath width is only comparable to its prediction until $\sim 3 R_{\odot}$. Our results also indicate a possible transition region around 3 to $3.5 R_{\odot}$ where the size of the sheath width exceeds its theoretical prediction, specially in the GL98 case. Since $W / R_{f r}>0.2$, we can say that both GL98 and TD99 CMEs sheath width are comparable to CMEs with propagation sheaths. However, in its very early stages the TD99 CME presents a sheath width size comparable to those presented by expansion sheaths.
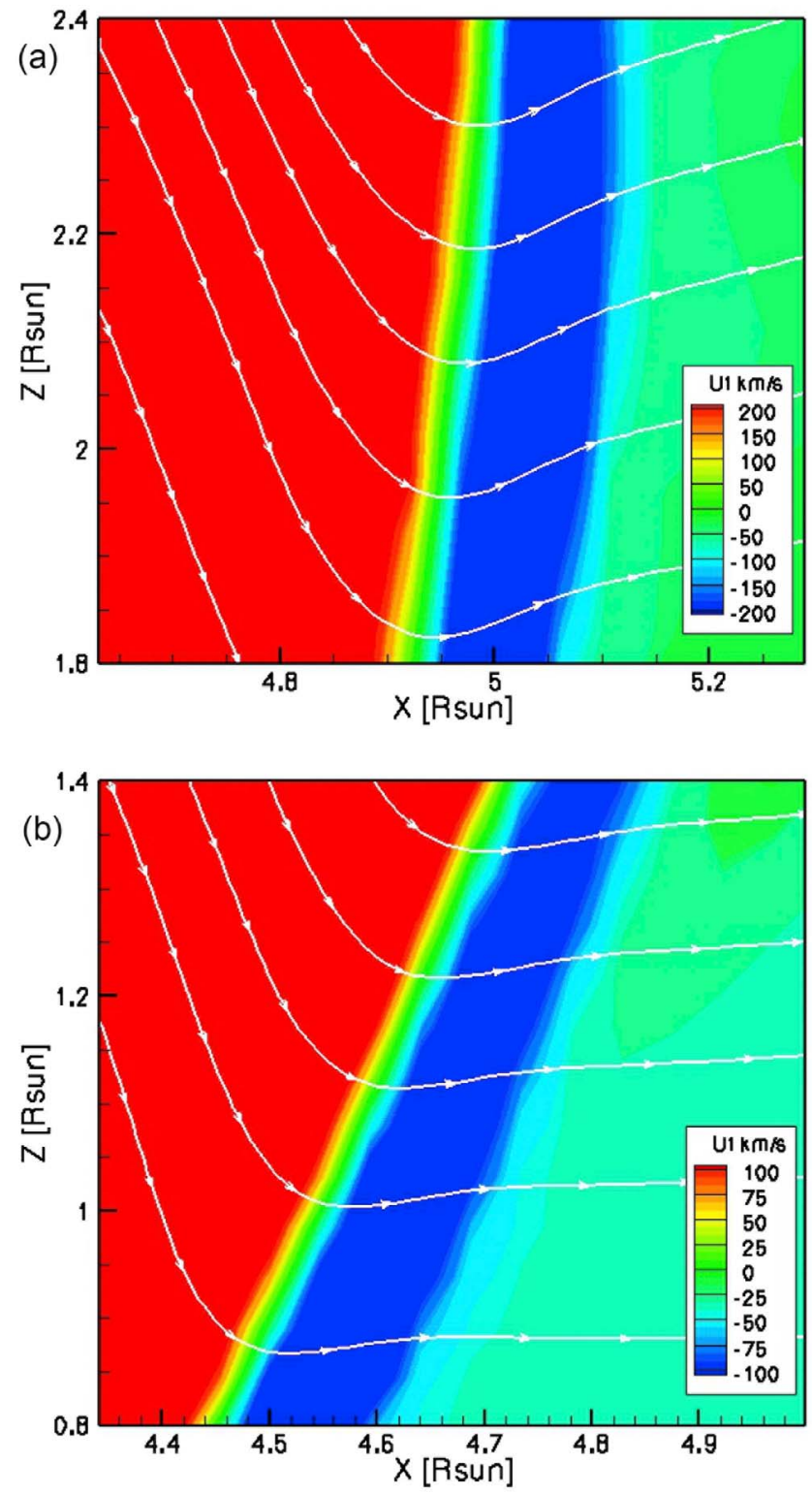

Figure 12. Meridional flow speed contour plot when the nose of the CME-driven shock is close to $5 R_{\odot}$ on the $\mathrm{x}-\mathrm{z}$ plane for (a) GL98 and (b) TD99. The white lines represent the magnetic field lines. Note that in both cases the field lines bend toward the equator at the shock front. 


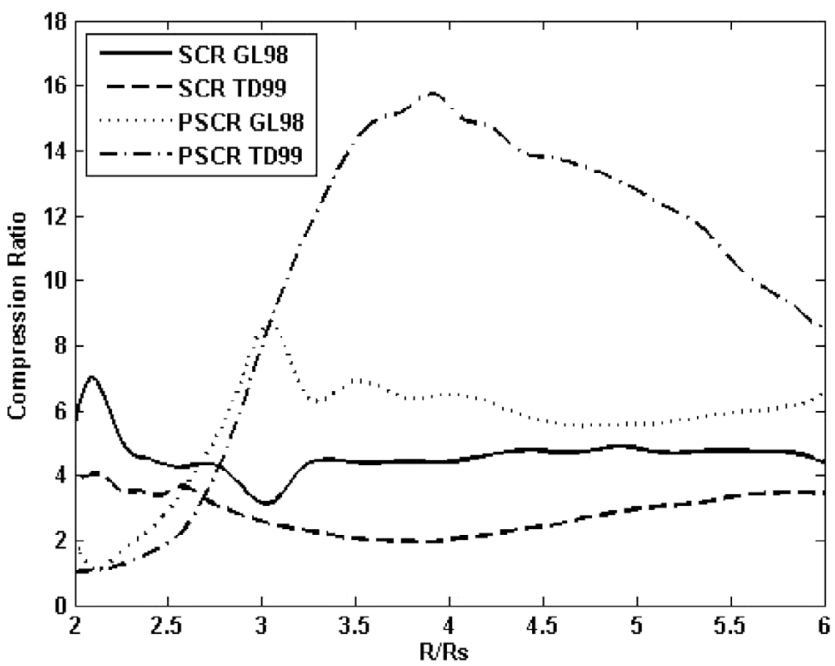

Figure 13. Shock compression ratio (SCR) and postshock compression ratio (PSCR) versus the shock position. The shock and postshock compression are shown as a solid line and a dotted line, respectively, for GL98 and as a dashed line and a dash-dotted line, respectively, for TD99. The PSCR was defined as the peak density in the CME sheath divided by the downstream density immediately behind the shock.

These results are consistent to those expected by Siscoe and Odstrcil [2008] for CMEs with a $\mathrm{M}_{\mathrm{A}}<4$.

[41] An important aspect of space weather is the acceleration of solar energetic particles (SEPs) at CME-driven shocks. SEPs can be accelerated within a short time after the CME initiation. Manchester et al. [2005] discussed the existence of a compression in the SW plasma behind the shock, the so-called postshock compression, that might contribute to the acceleration of SEPs. They identified this postshock compression as an increase in density and magnetic field strength behind the shock, where there is an indentation in the shock front. In this case, the postshock compression ratio was measured in the range of $6 R_{\odot}$ and $20 R_{\odot}$ although the compression extended to $1 \mathrm{AU}$. A similar postshock compression was described by Liu et al. [2008a] in the lower corona for $R \gtrsim 3 R_{\odot}$, using the TD99 model. From hereafter, we referred to this compression as a shock indentation compression (SIC).

[42] In work by Manchester et al. [2005] the existence of SIC is observed in their simulation of a GL98 CME. They reported that while the CME-driven shock propagates, away from the Sun, it interacts with the bimodal solar wind distorting its front, forming a dimple. A dimple is an indentation (concave-outward) in the shock front near the current sheet (observed in the $\mathrm{x}-\mathrm{z}$ plane), formed as a result of the difference in speed of the shock in high and low latitudes. Analyzing how flows are deflected at the dimple, they observed an enhancement in the density structure near the dimple due to a deflection of the flow from higher latitudes toward the equator. Such deflection produces a substantial compression behind the shock (postshock compression). They showed that SIC bent the field lines toward the equator and that there is a reversal in the direction of the flow in the sheath.

[43] Figure 12 presents the meridional flow speed $\left(U_{t}\right)$ for both the GL98 and TD99 CMEs when the nose of the shock is close to $5 R_{\odot}$. A postshock compression due to SIC is observed for both GL98 and TD99. We observe that the shock is bending the field lines toward the equator and that the flow reverses direction in the sheath. Our analysis have also shown an increase in the density in the sheath where the field lines bend to low latitude (toward the flux rope). Such results are in agreement to those results presented by Manchester et al. [2005]. Our results indicate a higher SIC for the GL98 CME than for the TD99 CME.

[44] Our analyses have also shown that the SIC and the shock compression are so spatially close that the density compressions may not be independently resolved, since they are separated by only 3 to 4 cells. In this case, the SIC and shock compressions may have merged into a single larger compression that is greater than the shock alone as was shown by Manchester et al. [2005]. The higher SIC for the GL98 CME and the merger between the SIC and the shock compressions may be contributing to GL98's larger compression ratio.

[45] Another compression in the sheath, shown in Figure 13, has been discussed by Das et al. [2011], which shows the formation of the pileup compression (PUC) for both the GL98 and TD99 CMEs. This compression is a consequence of the flux rope expansion and propagation speed being faster than the deflection flows of the SW in the CME sheath, just ahead of the flux rope. This is particularly true in the lower corona. Dasso et al. [2007] observed this solar wind material pileup just ahead of an interplanetary CME (ICME), when analyzing an event observed by the Wind spacecraft. Lynch et al. [2010], for example, observed plasma pileup structures around magnetic clouds.

[46] In Figure 13, we show that a postshock compression due to pileup effects (PUC) is observed for both the GL98 and TD99 CMEs for $R \gtrsim 2.5-3 R_{\odot}$. The postshock compression ratio, shown in Figure 13, was defined as the peak density in the CME sheath divided by the downstream density immediately behind the shock. The results show a much larger postshock compression ratio for the TD99 CME. Figure 13 also presents the shock compression ratio for both models. Our results show a much larger shock compression ratio for the GL98 model than for the TD99 model. The higher compression ratio for GL98 could be due to the higher magnetic energy and the higher thermal energy (due to sweeping of coronal material) as compared to the TD99 CME (see Figure 5). Another effect that could affect the GL98 shock compression ratio is the SIC.

[47] Besides the SIC and PUC postshock compressions caused by an increase in density in the sheath, Manchester et al. [2005] and Das et al. [2011] have suggested that a postshock compression may also be related to a plasma depletion layer (PDL). Contrary to what happens in the SIC and PUC postshock compressions, the PDL is characterized by a sharp density drop. They showed that a compression in the magnetic field lines occurs in the sheath where the plasma is rapidly expanding parallel to the magnetic field, while the field lines themselves are compressed by converging motion perpendicular to the magnetic field. The combined motions produce low density and high field strength in the sheath. A PDL was observed in the sheath by Liu et al. [2006] in front of a fast moving and expanding magnetic cloud, in the region where the magnetic field lines change around it. In this work we will not analyze PDL 


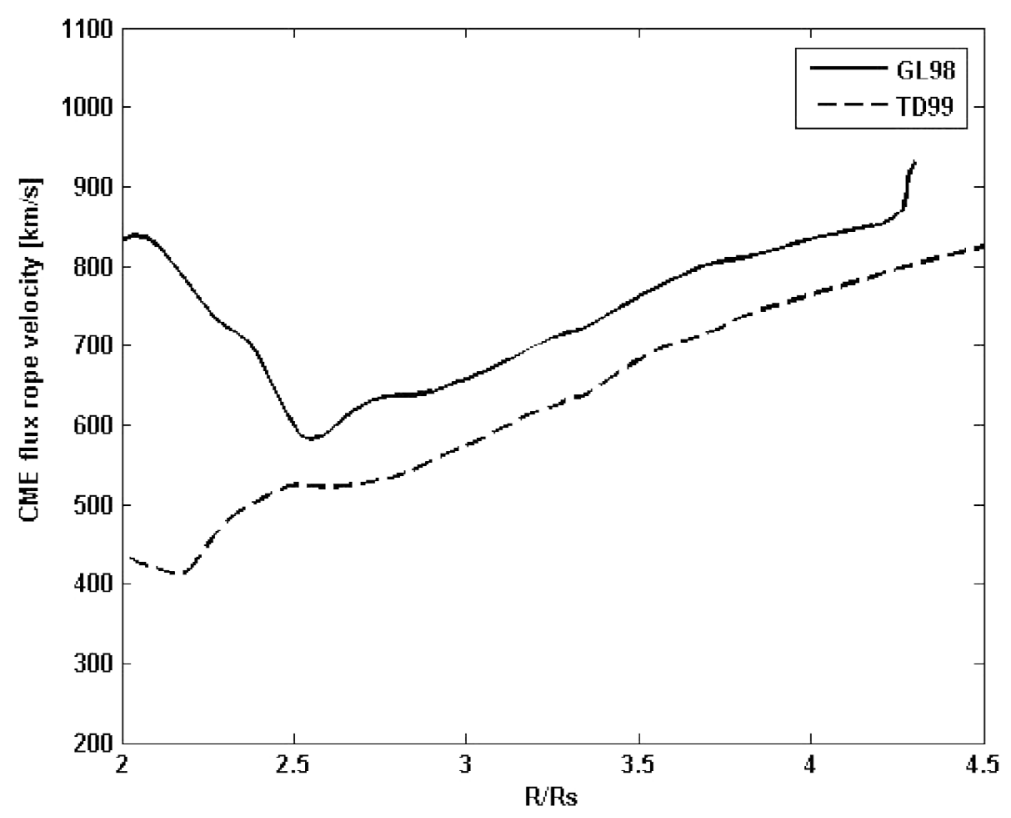

Figure 14. CME flux rope radial velocity for both driving mechanisms, GL98 (solid line) and TD99 (dashed line), calculated at the leading edge of the flux rope. These profiles do not account for the effect of the lateral expansion of the flux rope.

postshock compressions. A detailed discussion about the PUC and the effect of the PDL for GL98 and TD99 can be found in work by Das et al. [2011].

[48] Our results show that both the GL98 and TD99 CMEs present a compression in the sheath, called postshock compression (PSC). The PSC associated with the reversal of the flow in the region where the field lines bend to low latitude (toward the flux rope) and to an increase in density, we call SIC. The PSC observed to occur due to the pileup of the solar wind material ahead of the flux rope, we call PUC. Although, both SIC and PUC can be observed for the GL98 and TD99 CMEs, for GL98 CME the SIC is stronger while for the TD99 CME the PUC is stronger.

\subsection{CME Acceleration}

[49] Observations have shown that CMEs bulk of acceleration occurs near the Sun $\left(\sim 2-3 R_{\odot}\right)$ [MacQueen and Fisher, 1983 ] and that the maximum of acceleration typically occurs

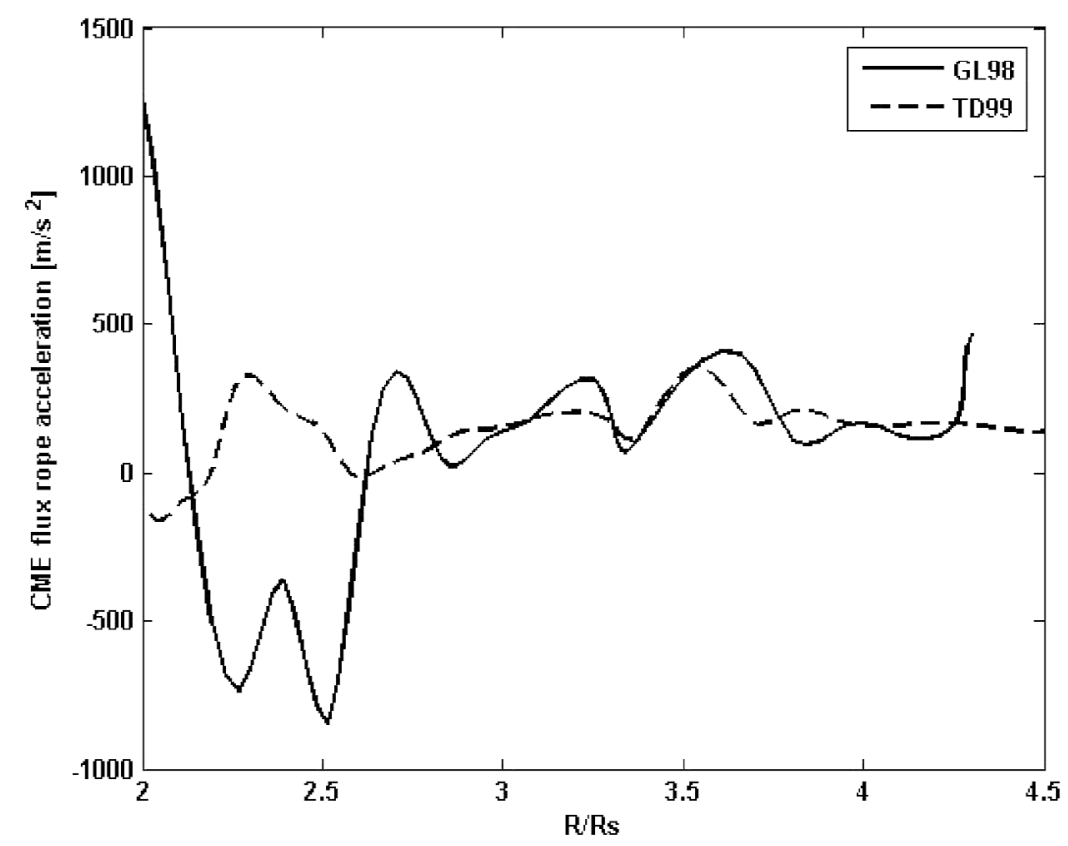

Figure 15. CME flux rope acceleration for both driving mechanisms, GL98 (solid line) and TD99 (dashed line). 
below $\sim 4 R_{\odot}$ [St. Cyr et al., 1999; Vrsnak, 2001]. Vrsnak et al. [1991] indicated that more twisted prominences have the peak of acceleration at lower heights. Close to the Sun, the forces acting on a flux rope can be represented by a combination of the Lorentz force $\left(F_{L}\right)$, gravity $\left(F_{G}\right)$, pressure $\left(F_{P}\right)$ and drag force $\left(F_{D}\right)$ [Howard et al., 2007]. The CME acceleration " $a_{C M E}$ " may be given by

$$
M \times a_{C M E}=F_{L}-\left(F_{G}+F_{P}+F_{D}\right),
$$

where $M$ can be considered as the sum of the CME mass $\left(M_{C M E}\right)$ and the virtual mass $\left(M_{V}\right)$ [Forbes et al., 2006]. The virtual mass is a concept from hydrodynamics related to the force needed to move away the ambient medium, as a body is accelerated in a fluid. In equation (3), the Lorentz force represents the curvature forces such as tension force exerted by the magnetic field lines [Krall and Trivelpiece, 1973; Spruit, 1981], and the hoop force, a particular form of the Lorentz force acting in curved, current-carrying plasmas [Chen, 1989, 1996; Chen and Krall, 2003]. $F_{P}$ represents the pressure gradient between the flux rope and the ambient coronal pressures. And, the last term on the right hand side of equation (3) is the drag force, due to the interaction between the CME and the ambient SW.

[50] As discussed above both the GL98 and the TD99 CMEs are initially driven by the magnetic energy (with an energy of an order of magnitude larger for the GL98 model), that is converted into thermal and kinetic energies as the CMEs propagate. Figure 14 presents the radial velocity $\left(\mathrm{U}_{\mathrm{fr}}\right)$ profiles for both CMEs flux ropes throughout the lower corona, where $\mathrm{U}_{\mathrm{fr}}=\left(\mathrm{U}_{\mathrm{x}} \cdot \mathrm{X}+\mathrm{U}_{\mathrm{y}} \cdot \mathrm{Y}+\mathrm{U}_{\mathrm{z}} \cdot \mathrm{Z}\right) /$ $\sqrt{\left(X^{2}+Y^{2}+Z^{2}\right)}$. GL98 and TD99 velocity profiles were calculated at the leading edge of the flux rope, and they do not account for the effect of the lateral expansion of the flux rope. Figure 14 shows that the GL98 flux rope has a higher radial velocity than the TD99 flux rope all the way to $5 R_{\odot}$. The CMEs flux rope velocity profiles are within the observed range [Zhang and Dere, 2006]. The acceleration profiles, presented in Figure 15, indicate that both CMEs flux ropes present similar accelerations for $R>3 R_{\odot}$. The average acceleration for the GL98 CME within 3 and $5 R_{\odot}$ is $\sim 207 \mathrm{~m} / \mathrm{s}^{2}$ and for TD99 is $\sim 192 \mathrm{~m} / \mathrm{s}^{2}$. In our simulations, the early acceleration of CMEs following an artificial insertion of a flux rope may not be physical. However, solutions, after $3 R_{\odot}$, have already settled down and reflect the total energy, mass and flux put in the system.

[51] As shown on equation (3), the forces acting on the flux rope close to the Sun have an important role in the CMEs acceleration. Our results indicate that the CME total pressure is in average twice larger for the GL98 model than for the TD99 model, until $\sim 6 R_{\odot}$. Analyzing both CMEs magnetic and thermal pressure, we observe that at the flux rope region, the magnetic pressure dominates the $\mathrm{CME}$ evolutions all the way to $\sim 6 R_{\odot}$. However, at the sheath the magnetic and thermal pressures have the same order of magnitude (see detailed analysis by Evans et al. [2011]). As expected, as the CMEs expand and propagate out of the Sun, the Lorentz force weakens and the drag force owing the solar wind becomes important [e.g., Cargill et al., 1996; Chen, 1996].
[52] These results indicate that differences in the initial magnetic configuration of two CMEs cannot be inferred from their acceleration profiles.

\section{Discussion and Conclusions}

[53] Here we performed a simulation with a grid refinement of $3 / 256 R_{\odot}$ during the CME propagation, finer than previous studies (for example, $1 / 32 R_{\odot}$ by Manchester et al. [2005, 2006] and 3/128 $R_{\odot}$ by Liu et al. [2008a]); we fixed the polytropic index distribution once the CME is initiated. The increased resolution served to minimize thermal heating due to reconnection. The polytropic index was set fixed to control the amount of heating in the system.

[54] We found that although we choose parameters to assure two CMEs with very different initial magnetic field configurations (with a highly twisted TD99 flux rope), the propagation of CMEs main observables is very similar in the lower corona. However, we believe through the analysis of other quantities, such as sheath width and postshock compression, the effect of different magnetic configurations and initializations can be distinguished.

[55] This paper indicates the importance of understanding the role of the magnetic field configuration in CMEs evolution close to the Sun and how difficult it might be to distinguish two different CME magnetic configurations by the profiles of their main observables in the lower corona. It also discusses the role of the background solar wind plays in the CMEs evolution.

[56] We found that both the GL98 and the TD99 CMEs are driven by their initial magnetic energy, that is converted into thermal and kinetic energies as the CMEs propagate. The remaining kinetic energies for the two cases differ due to the difference in mass between the two CMEs (bigger for the GL98 CME). We also found that the thermal energy is larger than the magnetic energy after $2 R_{\odot}$. We believe this happens due to the fact that the background solar wind $(\gamma<$ $5 / 3)$ is strongly heated as it propagates outward the Sun what may be affecting the CMEs evolution (for the estimation of the amount of heating see Evans et al. [2009]).

[57] Our results indicate that although the two CMEs possess different initial energies and magnetic configurations, the main observables such as acceleration, shock speed, Mach number, $\theta_{B n}$, etc, present very similar behavior between 2 and $6 R_{\odot}$.

[58] Comparing the results for the two models, we obtained the following.

[59] 1. The orientation of the angle between the shock normal and the upstream magnetic field $\left(\theta_{B n}\right)$ changes in the lower corona within 3 to $3.5 R_{\odot}$ from the Sun, for both models; At the nose, the shocks are quasi-perpendicular for distances $<3 R_{\odot}$ from the Sun. This is due partially because at earlier times the CMEs are within the coronal streamer.

[60] 2. The CME shock speeds are very similar for both models, being slightly higher for the GL98 model due to the higher magnetic field in its flux rope, in agreement with the results by Manchester et al. [2008].

[61] 3. Both models present a postshock compression for $R>2.5 R_{\odot}$, stronger for TD99. Both the GL98 and TD99 CMEs present postshock compressions related to a shock indentation compression (SCI) associated to the reversal of 
the flow in the region where the field lines bend to low latitude (toward the flux rope) and an increase in the density. Similarly, both CMEs present a postshock compressions caused by a high-density pileup compression (PUC) due to the solar wind material being pushed ahead of the flux rope.

[62] 4. The general profiles of the CMEs Mach number are very similar for both models, with larger valued $\mathrm{M}_{\mathrm{A}}$ for GL98 than for TD99. There is a decrease for $\mathrm{M}_{\mathrm{A}}\left(R>4 R_{\odot}\right)$ for the TD99 model once the shock speed starts to decrease (as in work by Manchester et al. [2005]). While the CME propagates material is swept up by it and deceleration happens due to momentum conservation. However, the fact that TD99 magnetic energy dissipates quickly due to numerical reconnection could also play a role decelerating the shock. We attempt to minimize the magnetic dissipation with a high-resolution grid at the nose. GL98 presents a higher compression due to its higher magnetic energy and the higher thermal energy(due to sweeping of solar material). GL98 CME shock compression can also be related to its faster shock speed, its high Mach number and, also, due to its more rapid expansion than the TD99 CME. This indicates that it should accelerate particles more efficiently than the TD99 model. However, the TD99 CME shows a larger postshock compression ratio, which could contribute to acceleration of particles as well.

[63] 5. The GL98 CME sheath is almost twice larger than the TD99 CME one. Both CMEs present sheath width sizes $W / R_{f r}>0.2$, being comparable to CMEs with propagation sheaths. The size of both CMEs sheath width is consistent to what is expected for CMEs with a $\mathrm{M}_{\mathrm{A}}<4$ [e.g., Siscoe and Odstrcil, 2008].

[64] 6. We have found that the CME flux rope acceleration profiles for both models is similar for $R>3 R_{\odot}$. The average acceleration is: $\sim 207 \mathrm{~m} / \mathrm{s}^{2}$ for the GL98 model and $\sim 192 \mathrm{~m} / \mathrm{s}^{2}$ for the TD99 model; These accelerations are within the observed range [Zhang and Dere, 2006].

[65] Acknowledgments. The simulations presented here were performed at the NASA/Ames Columbia supercomputers. M.O. acknowledges the support by National Science Foundation CAREER grant ATM0747654 and NASA Ames. M.V.A. is thankful for the support given by the Brazilian agency CNPq. W.B.M. acknowledges the support given by LWS grant NNX09AJ78G. C.L. would like to thank César A. Costa, Aline Vidotto, and Yong Liu for their cooperation and for valuable discussions during this project.

[66] Philippa Browning thanks the reviewers for their assistance in evaluating this paper.

\section{References}

Antiochos, S. K., C. R. DeVore, and J. A. Klimchuk (1999), A model for solar coronal mass ejections, Astrophys. J., 510, 485-493.

Arge, C. N., and V. J. Pizzo (2000), Improvement in the prediction of solar wind conditions using near-real time solar magnetic field updates, J. Geophys. Res., 105, 10465-10480, doi:10.1029/1999JA900262.

Arge, C. N., J. G. Luhmann, D. Odstrcil, C. J. Schrijver, and Y. Li (2004), Stream structure and coronal sources of the solar wind during the May 12th, 1997 CME, J. Atmos. Sol. Terr. Phys., 66(15-16), 1295-1309, doi:10.1016/j.jastp.2004.03.018.

Burlaga, L. F. (1988), Magnetic clouds and force-free fields with constant alpha, J. Geophys. Res., 93, 7217-7224, doi:10.1029/JA093iA07p07217.

Cane, H. V., N. R. Sheeley, and R. A. Howard (1987), Energetic interplanetary shocks, radio emission, and coronal mass ejections, J. Geophys. Res., 92, 9869-9874, doi:10.1029/JA092iA09p09869.

Cargill, P. J., J. Chen, D. S. Spicer, and S. T. Zalesak (1996), Magnetohydrodynamic simulations of the motion of magnetic flux tubes through a magnetized plasma, J. Geophys. Res., 101, 4855-4870, doi:10.1029/ 95JA03769.

Chen, J. (1989), Effects of toroidal forces in current loops embedded in a background plasma, Astrophys. J., 338, 453-470, doi:10.1086/167211.

Chen, J. (1996), Theory of prominence eruption and propagation: Interplanetary consequences, J. Geophys. Res., 101, 27,499-27,520, doi:10.1029/ 96JA02644.

Chen, J., and J. Krall (2003), Acceleration of coronal mass ejections, J. Geophys. Res., 108(A11), 1410, doi:10.1029/2003JA009849.

Cohen, O., et al. (2007), A semiempirical magnetohydrodynamical model of the solar wind, Astrophys. J., 654, L163-L166, doi:10.1086/511154.

Cohen, O., I. V. Sokolov, I. I. Roussev, and T. I. Gombosi (2008), Validation of a synoptic solar wind model, J. Geophys. Res., 113, A03104, doi:10.1029/2007JA012797.

Das, I., M. Opher, R. M. Evans, C. Loesch, and T. I. Gombosi (2011), Evolution of piled up compressions in modeled CME sheaths and the resulting sheath structures, Astrophys. J., 729, 112-118, doi:10.1088/0004-637X/ $729 / 112$

Dasso, S., M. S. Nakwacki, P. Demoulin, and C. H. Mandrini (2007), Progressive transformation of a flux rope to an ICME. Comparative analysis using the direct and fitted expansion methods, Sol. Phys., 244, 115-137, doi:10.1007/s11207-007-9034-2.

Evans, R. M., M. Opher, V. Jatenco-Pereira, and T. I. Gombosi (2009), Surface Alfvén wave damping in a 3D simulation of the solar wind, $A s-$ trophys. J., 730, 179-186, doi:10.1088/0004-637X/703/1/179.

Evans, R. M., M. Opher, and T. I. Gombosi (2011), Learning from the outer heliosphere: Interplanetary coronal mass ejection sheath flows and the ejecta orientation in the lower corona, Astrophys. J., 728, 41-47, doi:10.1088/0004-637X/1/41.

Forbes, T. G., and E. R. Priest (1995), Photospheric magnetic field evolution and eruptive flares, Astrophys. J., 446, 377-389, doi:10.1086/ 175797.

Forbes, T. G., et al. (2006), CME theory and models, Space Sci. Rev., 123(1-3), 251-302, doi:10.1007/s11214-006-9019-8.

Gibson, S. E., and B. C. Low (1998), A time-dependent three-dimensional magnetohydrodynamic model of the coronal mass ejection, Astrophys. $J ., 493,460-473$, doi:10.1086/305107.

Howard, T. A., C. D. Fry, J. C. Johnston, and D. F. Webb (2007), On the evolution of coronal mass ejections in the interplanetary medium, Astrophys. J., 667, 610-625, doi:10.1086/519758.

Hundhausen, A. J. (1997), An introduction, in Coronal Mass Ejections, edited by N. Crooker, J. Joselyn, and J. Feyman, pp. 1-7, AGU, Washington, D. C.

Jacobs, C., I. I. Roussev, N. Lugaz, and S. Poedts (2009), The internal structure of coronal mass ejections: Are all regular magnetic clouds flux ropes?, Astrophys. J., 695, L171-L175, doi:10.1088/0004-637X 695/2/L171.

Kivelson, M. G., and C. T. Russel (Eds.) (1995), Introduction to Space Physics, 1st ed., 568 pp., Cambridge Univ. Press, New York.

Krall, N. A., and A. W. Trivelpiece (Eds.) (1973), Principles of Plasma Physics, 1st ed., 568 pp., McGraw-Hill, Tokyo.

Le, G. M., and Y. B. Han (2005), Analysis of the acceleration process of SEPs by an interplanetary shock for Bastille Day event, in Coronal and Stellar Mass Ejections: Proceedings of the IAU Symposium 226 , edited by K. Dere, J. Wang, and Y. Yan, pp. 346-349, Cambridge Univ. Press, Cambridge, U. K.

Linker, J. A., and Z. Mikić (1995), Disruption of a helmet streamer by photospheric shear, Astrophys. J., 438, L45-L48, doi:10.1086/187711.

Liu, Y., J. D. Richardson, J. W. Belcher, J. C. Kasper, and R. M. Skoug (2006), Plasma depletion and mirror waves ahead of interplanetary coronal mass ejections, J. Geophys. Res., 111, A09108, doi:10.1029/ 2006JA011723.

Liu, Y. C.-M., M. Opher, O. Cohen, P. C. Liewer, and T. I. Gombosi (2008a), A simulation of a coronal mass ejection propagation and shock evolution in the lower solar corona, Astrophys. J., 680, 757-763, doi: $10.1086 / 587867$.

Liu, Y., W. B. Manchester, J. D. Richardson, J. G. Luhmann, R. P. Lin, and S. D. Bale (2008b), Deflection flows ahead of ICMEs as an indicator of curvature and geoeffectiveness, J. Geophys. Res., 113, A00B03, doi:10.1029/2007JA012996.

Lugaz, N., W. B. Manchester, I. I. Roussev, G. Tóth, and T. I. Gombosi (2007), Numerical investigation of the homologous coronal mass ejection events from active region 9236, Astrophys. J., 659, 788-800, doi: $10.1086 / 512005$.

Lugaz, N., A. Vourlidas, I. I. Roussev, and H. Morgan (2009), Solar-terrestrial simulation in the STEREO era: The 24-25 January 2007 eruptions, Sol. Phys., 256, 269-284, doi:10.1007/s11207-009-9339-4.

Lynch, B. J., Y. Li, A. F. R. Thernisien, E. Robbrecht, G. H. Fisher, J. G. Luhmann, and A. Vourlidas (2010), Sun to $1 \mathrm{AU}$ propagation and 
evolution of a slow streamer-blowout coronal mass ejection, J. Geophys. Res., 115, A07106, doi:10.1029/2009JA015099.

MacQueen, R. M., and R. R. Fisher (1983), The kinematics of solar inner coronal transients, Sol. Phys., 89, 89-102, doi:10.1007/BF00211955.

Manchester, W. (2003), Buoyant disruption of magnetic arcades with selfinduced shearing, J. Geophys. Res., 108(A4), 1162, doi:10.1029/ 2002JA009252.

Manchester, W. (2007), Solar atmospheric dynamic coupling due to shear motions driven by the Lorentz force, Astrophys. J., 666, 532-540, doi:10.1086/520493.

Manchester, W., T. I. Gombosi, I. Roussev, D. L. De Zeeuw, I. V. Sokolov, K. G. Powell, G. Tóth, and M. Opher (2004a), Three-dimensional MHD simulation of a flux rope driven CME, J. Geophys. Res., 109, A01102, doi:10.1029/2002JA009672.

Manchester, W. B., T. I. Gombosi, I. Roussev, A. Ridley, D. L. De Zeeuw, I. V. Sokolov, K. G. Powell, and G. Tóth (2004b), Modeling a space weather event from the Sun to the Earth: CME generation and interplanetary propagation, J. Geophys. Res., 109, A02107, doi:10.1029/ 2003JA010150.

Manchester, W. B., T. I. Gombosi, D. L. De Zeeuw, and Y. Fan (2004c), Eruption of a buoyantly emerging magnetic flux rope, Astrophys. J., 610 , 588-596, doi:10.1086/421516.

Manchester, W. B., T. I. Gombosi, D. L. De Zeeuw, I. V. Sokolov, I. I. Roussev, K. G. Powell, J. Kóta, G. Tóth, and T. H. Zurbuchen (2005), Coronal mass ejection shock and sheath structures relevant to particle acceleration, Astrophys. J., 622, 1225-1239, doi:10.1086/427768.

Manchester, W. B., A. J. Ridley, T. I. Gombosi, and D. L. De Zeeuw (2006), Modeling the Sun-to-Earth propagation of a very fast CME, Adv. Space Res., 38(2), 253-262, doi:10.1016/j.asr.2005.09.044.

Manchester, W. B., A. Vourlidas, T. Gábor, N. Lugaz, I. I. Roussev, I. V. Sokolov, T. I. Gombosi, D. L. De Zeeuw, and M. Opher (2008), Threedimensional MHD simulation of the 2003 October 28 coronal mass ejection: Comparison with LASCO coronagraph observations, Astrophys. J., 684, 1448-1460, doi:10.1086/590231.

Mancuso, S., and A. Bemporad (2009), Interpretation of the SOHO/UVCS observations of two CME-driven shocks, Adv. Space Res., 44(4), 451-456, doi:10.1016/j.asr.2009.03.021.

Odstrcil, D., J. A. Linker, R. Lionello, Z. Mikić, P. Riley, V. J. Pizzo, and J. G. Luhmann (2002), Merging of coronal and heliospheric numerical two-dimensional MHD models, J. Geophys. Res., 107(A12), 1493, doi:10.1029/2002JA009334.

Roussev, I. I., T. G. Forbes, T. I. Gombosi, I. V. Sokolov, D. L. De Zeeuw, and J. Birn (2003a), A three-dimensional flux rope model for coronal mass ejections based on a loss of equilibrium, Astrophys. J., 588, L45-L48, doi:10.1086/375442.

Roussev, I. I., T. I. Gombosi, I. V. Sokolov, M. Velli, W. Manchester IV, D. L. Dezeeuw, P. Liewer, G. Tóth, and J. Luhmann (2003b), A threedimensional model of the solar wind incorporating solar magnetogram observations, Astrophys. J., 595, L57-L61, doi:10.1086/378878.

Roussev, I. I., I. V. Sokolov, T. G. Forbes, T. I. Gombosi, M. A. Lee, and J. I. Sakai (2004), A numerical model of a coronal mass ejection: Shock development with implications for the acceleration of $\mathrm{GeV}$ protons, Astrophys. J., 605, L73-L76, doi:10.1086/392504.

Sheeley, N. R., Jr., R. A. Howard, D. J. Michels, M. J. Koomen, R. Schwenn, K. H. Muehlhaeuser, and H. Rosenbauer (1985), Coronal mass ejections and interplanetary shocks, J. Geophys. Res., 90, 163-175, doi:10.1029/JA090iA01p00163.

Siscoe, G., and D. Odstrcil (2008), Ways in which ICME sheaths differ from magnetosheaths, J. Geophys. Res., 113, A00B07, doi:10.1029/ 2008JA013142.
Spruit, H. C. (1981), Motion of magnetic flux tubes in the solar convection zone and chromosphere, Astron. Astrophys., 98, 155-160.

St. Cyr, O. C., J. T. Burkepile, A. J. Hundhausen, and A. R. Lecinski (1999), A comparison of ground-based and spacecraft observations of coronal mass ejections from 1980-1989, J. Geophys. Res., 104, 12,493-12,506, doi:10.1029/1999JA900045.

Titov, V. S., and P. Démoulin (1999), Basic topology of twisted magnetic configuration in solar flares, Astron. Astrophys., 351, 707-720.

Torok, T., and B. Kliem (2005), Confined and ejective eruptions of kinkunstable flux ropes, Astrophys. J., 630, L97-L100, doi:10.1086/462412.

Tóth, G., et al. (2005), Space weather modeling framework: A new tool for the space science community, J. Geophys. Res., 110, A12226, doi:10.1029/2005JA011126.

Tsurutani, B. T., W. D. Gonzalez, F. Tang, S.-I. Akasofu, and E. J. Smith (1988), Origin of interplanetary southward magnetic fields responsible for major magnetic storms near solar maximum (1978-1979), J. Geophys. Res., 93, 8591-8531, doi:10.1029/JA093iA08p08519.

Vourlidas, A., D. Buzasi, R. A. Howard, and E. Esfandiari (2002), Mass and energy properties of LASCO CMEs, in Solar Variability: From Core to Outer Frontiers, vol. 1, edited by A. Wilson, pp. 91-94, Eur. Space Agency, Noordwijk, Netherlands.

Vrsnak, B. (2001), Dynamics of solar coronal eruptions, J. Geophys. Res., 106, 25,249-25,260, doi:10.1029/2000JA004007.

Vrsnak, B., V. Ruzdjak, and B. Rompolt (1991), Stability of prominences exposing helical-like patterns, Sol. Phys., 136, 151-167, doi:10.1007/ BF00151701.

Wang, R., and J. Wang (2006), Spectra and solar energetic protons over $20 \mathrm{GeV}$ in Bastille Day event, Astroparticle Phys., 25(1), 41-46, doi:10.1016/j.astropartphys.2005.11.002.

Watari, S., and T. Detman (1998), In situ local shock speed and transit shock speed, Ann. Geophys., 16(4), 370-375, doi:10.1007/ s005850050607.

Wu, S. T., W. P. Guo, D. J. Michels, and L. F. Burlaga (1999), MHD description of the dynamical relationships between a flux rope, streamer, coronal mass ejection, and magnetic cloud: An analysis of the January 1997 Sun-Earth connection event, J. Geophys. Res., 104, 14,789-14,802, doi:10.1029/1999JA900099.

Wu, S. T., M. D. Andrews, and S. P. Plunkett (2001), Numerical magnetohydrodynamic (MHD) modeling of coronal mass ejections (CMEs), Space Sci. Rev., 95(1-2), 191-213.

Zhang, J., and K. P. Dere (2006), A statistical study of main and residual accelerations of coronal mass ejections, Astrophys. J., 649, 1100-1109, doi:10.1086/506903.

Zhang, J., W. Poomvises, and I. G. Richardson (2008), Sizes and relative geoeffectiveness of interplanetary coronal mass ejections and the preceding shock sheaths during intense storms in 1996-2005, Geophys. Res. Lett., 35, L02109, doi:10.1029/2007GL032045.

M. V. Alves and C. Loesch, Laboratório Associado de Plasma, Instituto Nacional de Pesquisas Espaciais, Avenida dos Astronautas 1758, Jardim da Granja, São José dos Campos, 12227-010, Brazil. (virginia@plasma.inpe. br; crisloesch@gmail.com)

R. M. Evans and M. Opher, Department of Physics and Astronomy, George Mason University, Sci and Tech I MSN 3F3, 4400 University Dr., Fairfax, VA 22030, USA. (revansa@gmu.edu; mopher@physics. gmu.edu)

W. B. Manchester, Center for Space Environment Modeling, University of Michigan, 2455 Hayward St., Ann Arbor, MI 48108, USA. (chipm@ umich.edu) 